\title{
Measurement and compensation of printer modulation transfer function
}

\author{
Nicolas Bonnier* \\ Albrecht J. Lindner* \\ Océ Print Logic Technologies \\ 1 rue Jean Lemoine \\ Créteil, 94015 France \\ and \\ Telecom ParisTech \\ Institut Telecom \\ CNRS UMR 5141 LTCI \\ 75013 Paris, France \\ E-mail: nicolas.bonnier@oce.com \\ E-mail: albrecht.lindner@epfl.ch
}

\begin{abstract}
The capacity of a printing system to accurately reproduce details has an impact on the quality of printed images. The ability of a system to reproduce details is captured in its modulation transfer function (MTF). In the first part of this work, we compare three existing methods to measure the MTF of a printing system. After a thorough investigation, we select the method from Jang and Allebach and propose to modify it. We demonstrate that our proposed modification improves the measurement precision and the simplicity of implementation. Then we discuss the advantages and drawbacks of the different methods depending on the intended usage of the MTF and why Jang and Allebach's method best matches our needs. In the second part, we propose to improve the quality of printed images by compensating for the MTF of the printing system. The MTF is adaptively compensated in the Fourier domain, depending both on frequency and local mean values. Results of a category judgment experiment show significant improvement as the printed MTF-compensated images obtain the best scores. (C) 2010 SPIE and IS\&T. [DOI: 10.1117/1.3272958]
\end{abstract}

\section{Introduction}

In the quest for optimal reproduction of color images, preservation of the spatial details in images has emerged as a very important issue for perceptual quality. ${ }^{1,2}$ To achieve high-quality reproduction, output devices should be able to reproduce adequately all the colors and details (highfrequency content) in the processed images. Thus, with the growing demand for high-quality reproduction of photographic images using inkjet printers, the interest in qualifying and quantifying the behavior of printing systems has become more and more important.

The ability of a system to reproduce details is captured in its modulation transfer function (MTF). Our goal is to obtain a robust characterization method for printing sys-

\footnotetext{
*This work was carried out under supervision of the late Pr. Francis Schmitt. Both authors have put equal amounts of work in this article and thus have been listed in alphabetical order.

Paper 09065SSR received May 1, 2009; revised manuscript received Aug. 12, 2009; accepted for publication Sep. 17, 2009; published online Jan. 13, 2010 .
}

1017-9909/2010/19(1)/011010/22/\$25.00 @ 2010 SPIE and IS\&T. tems. More precisely, we want to characterize it by its MTF. Strictly speaking, we do not measure the MTF but the spatial frequency response (SFR). The MTF is coming from optics and is measured for continuous frequencies, whereas the SFR is measured for quantized frequency values. Nevertheless, in this work we keep the term MTF as previously found in the literature. ${ }^{3-5}$ The measure of the MTF is based on the assumption that the printer is a linear system. A printer MTF describes how much a sinusoidal signal at the input (digital file) is attenuated by the system at the output (printout) for each spatial frequency $f$ [see Eq. (1)]. It is necessary to highlight the different representations at the input and output, since establishing a relation between a digital signal and a signal printed on paper is not evident.

Characterization of the MTF has been popular in photography, and there are a few methods to measure the MTF for both silver-halide and digital image capture devices, in which sinusoidal and slanted-edge targets are used. Some of these characterization methods have been standardized by the International Organization for Standardization (ISO). ${ }^{6,7}$ While much effort has been spent on the characterization of the color characteristic curves of printing systems, until recently little research ${ }^{3,4,8,9}$ has been published regarding the characterization of the MTF of printing systems. Arney et al. ${ }^{8}$ proposed a method to measure the MTF of the physical printing process (without the software preprocessing), by dividing the spectrum of the printed image by the spectrum of the halftoned image. Jang and Allebach ${ }^{4}$ proposed a procedure for characterizing the MTF of a complete digital printing system, i.e., the software, hardware, and the medium, in which test pages consisting of series of patches with different 1-D sinusoidal modulation are printed, scanned, and analyzed (see Sec. 5). Later, Hasegawa et $\mathrm{al}^{3}{ }^{3}$ proposed an objective metric to evaluate the quality of the resolution of a black and white laser printer. Similar to Jang and Allebach, black and white stripes, lines, and dot patterns are printed, scanned, and analyzed, yet the analyses in the two methods are very different. In Hase- 
gawa et al. the analysis is done by comparing black and white surfaces in the input image versus the corresponding surfaces on the printout, thus it is mainly a measure of the dot gain. Recently, the slanted-edge method used to characterize scanners and cameras ${ }^{7}$ has been adapted by Bang, $\mathrm{Kim}$, and $\mathrm{Choi}^{9}$ to measure the MTF of a printer: an image containing a slanted black square on white background is sent to the printer, then the printout is scanned and analyzed.

In this work we explore and compare the methods proposed by Jang and Allebach, ${ }^{4}$ by Hasegawa et al., ${ }^{3}$ and the slanted edge method adapted to printing systems by Bang, Kin, and Choi. ${ }^{9}$ Section 2 begins with a survey of the parameters a printer MTF might depend on to select an appropriate subset out of them that sufficiently describes the printer behavior. Then the experimental measuring setup used for all the experiments is described. In Sec. 3 the slanted edge method is presented, and is implemented as proposed in Refs. 7 and 9. Section 4 is dedicated to the exploration of the method proposed by Hasegawa et al. ${ }^{3}$ After introducing this method, we propose a simplification for the hypothesis test of the statistical analysis.

Furthermore, we point out the importance of the scan resolution for the hypothesis test, since it influences strongly the test and should be chosen consciously. In Sec. 5 the approach proposed by Jang and Allebach ${ }^{4}$ is explored and modifications are proposed primarily to reduce noise in the measurement. We found that this can be achieved by increasing the amplitudes of the test patches. Finally, the three methods are compared to each other and the results are discussed in Sec. 6.

In the second part of this work, we propose to compensate for the MTF of printing systems to enhance the quality of the output images. In Sec. 7 we consider the use of existing filters to compensate for the MTF of a printing system. In Sec. 8 we present a new locally adaptive method to compensate for the printer MTF. In Sec. 9 we compare the proposed algorithm to existing alternatives, then discuss possible evolutions. Then in Sec. 10 we explore the concept of overcompensating the MTF. The compensation algorithm is tested (Sec. 11) in a psychophysical experiment with photographic images to test its impact on the visual appearance of images. The results of this category judgment experiment are presented and commented. The newly developed algorithm shows very good results: the printer MTF is well compensated and no visual artifacts are introduced in the images. A final conclusion is given in Sec. 12 .

\section{Setup for the Experimental Evaluation of the Printer Modulation Transfer Function}

\subsection{Printer Modulation Transfer Function}

The MTF of a given system shows how much the system attenuates an input modulation signal. When considering a 1-D spatial modulation, the MTF is defined as the ratio of the output amplitude $A_{\text {out }}(f)$ to the input amplitude $A_{\text {in }}(f)$ :

$\operatorname{MTF}(f)=\frac{A_{\text {out }}(f)}{A_{\text {in }}(f)}$,

where $f$ is the spatial frequency of the 1-D signal (see Fig. $1)$.

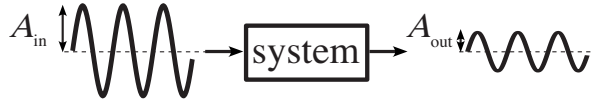

Fig. 1 Attenuation of a 1-D input sinusoidal signal with amplitude $A_{\text {in }}$ by a system resulting in a reduced output amplitude $A_{\text {out }}$.

An ideal imaging device would have an MTF equal to 1 for every frequency and orientation. Usually a printing system has low-pass characteristics: mostly only high frequencies are attenuated and low frequencies are preserved.

In preliminary experiments, ${ }^{10}$ we found that the MTF of our printing system was much lower than the MTF of our monitor. The technologies are indeed very different, and the lower MTF of the printing system is mainly due to the halftoning algorithm and to the ink spreading on the paper.

Characterizing the MTF of a printing system is not a trivial task: unlike digital capture devices, inkjet and laser printers are highly nonlinear and therefore not easy to model. The MTF depends on different parameters related to the printing system (halftoning, addressability, dot size, quality of the medium) and on the characteristics of the input image (bias, color, spatial frequency, orientation). This is also related to the Nyquist-Shannon sampling theorem. ${ }^{11}$ To fully characterize or describe a printing system, the MTF has to be measured for different biases, colors, frequencies, or orientations (see Sec. 2.3). For a modulation signal at a given set of bias, color, and orientations, the results can be plotted in a graph as in Fig. 2, where the MTF is plotted versus the frequency.

\subsection{How to Measure a Printer Modulation Transfer Function}

The basics described in Sec. 2.1 do not entirely cover the complexity of the MTF of a printing system. First, we are reminded a trivial fact: the output is a 2-D signal (image) printed on a media, and thus the MTF of the system depends on two perpendicular dimensions. More importantly, this printout approximates a pseudocontinuous input signal (such as an 8-bit grayscale image) with an halftoned image (typically a binary 1 - bit image) composed of dots on a grid pattern, whereas the definition of an MTF is based on the assumption that both the input and output signals are continuous in space and value. Neither of these assumptions is true for a printout: the signal is not continuous in space,

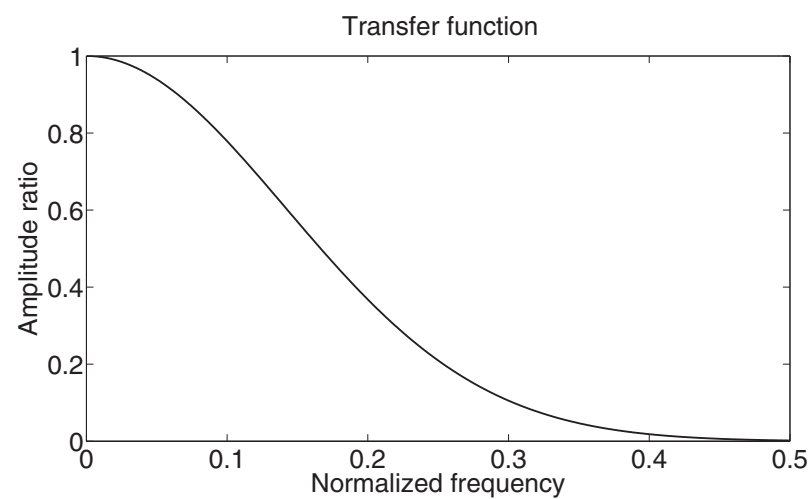

Fig. 2 Example of a modulation transfer function of a hypothetical system with low-pass characteristics. 
since the base of the signal is a grid with finite resolution and the signal is not continuous in value, since a grid cell only contains a binary information (dot or no-dot). Nevertheless, the printed signal appears to be continuous in space and value for an observer with normal viewing distance. This is because the grid is too fine and the observer's visual system blurs it into a quasicontinuous signal.

On the one hand a method to measure a printer MTF should be objective and based on physical properties so that anyone can reproduce results and compare them to others. On the other hand, such a method has to account for the fact that printing dots on a grid structure only makes sense if one assumes that an observer gets a continuous impression due to spatial blurring. A method to measure a printer MTF has to satisfy both requirements.

\subsection{Test Images}

For a fixed complete printing system, an MTF can depend on different parameters related to the characteristics of the input images. Among them are:

- bias and amplitude of spatially periodic changes

- color

- direction of spatial modulations or contours (horizontal, vertical, or intermediate angles)

- content of pixels printed just before (printed value could depend on previous print head actions)

- spatial position (a sequence of pixels could depend on its position on the paper)

The bias level should be considered, since the halftoning process and ink flow on the paper depend on it (e.g., through the dot gain control). Under the assumption of a linear system, the amplitude should not matter. Unfortunately, this is not absolutely true and is discussed in Sec. 5.3. The choice of the direction in which an MTF should be measured is important, since the complexity of the measurement could be significantly reduced. The physical characteristics of inkjet printers (with perpendicular slow and fast print directions) lead naturally to the decision to start with a measure in these two directions. For reasons of simplicity, we consider the printer as having no memory effect and as being independent of the spatial position. Additionally, we limit to the case with gray level images, since this work discusses more basic effects where color plays a secondary role.

\subsection{Printing Systems}

The characterization methods studied here can be performed on any kind of printing system. But to be able to analyze and compare results obtained by the different methods, it is necessary to choose and keep a fixed printing system for the whole evaluation. The printing system used for the comparison of the three methods is an Océ TCS-500 (Océ, Venlo, The Netherlands) large format inkjet printer with standard Océ uncoated paper at $600 \mathrm{dpi}$. The modifications to improve Jang and Allebach's method have been carried out on an Océ Colorwave 600 on Océ Red Label paper at $600 \mathrm{dpi}$. The latter printing system has also been used in the compensation of the MTF (see Secs. 7-11). For both printers, the ink values are coded on 8 bits and only the black ink has been used (gray level mode). However, the compensation is done on color images.

If dot gain has an impact on the printed color values, an initial linearization has to be achieved. ${ }^{12}$ Since the analysis is based on a physical measurement, it is reasonable to use the CIE XYZ space, and we decided to linearize our printer with a luminance scale according to the measured $Y$ values.

The test images are generated using $Y$ values that lie in the printable interval $\left[Y_{\text {low }}, Y_{\text {high }}\right]$ and are then converted to numeric ink levels in the interval $[0 . .255]$ with the help of a look-up table (LUT). In our experiment, $Y_{\text {low }}=17.8$ and $Y_{\text {high }}=85.6$.

\subsection{Scanner}

The scanner used for the analysis is an Epson Expression 10000XL (Seiko Epson Corp., Nagano, Japan), which has a much higher native resolution than the printer (up to $2400 \mathrm{dpi}$ ). In our experiments we set the scanning resolution at $1200 \mathrm{dpi}$. This scanning resolution, two times higher than the printing resolution, is necessary to avoid aliasing problems when scanning the printout to measure the MTF.

Since a scanner has itself a degrading influence, it must be compensated for in the analysis. Therefore the MTF of the scanner has been measured with the slanted edge method following the standard ISO 16067-1. ${ }^{7}$

Jang and Allebach ${ }^{4}$ propose to compensate the scanner MTF to obtain the MTF of only the printing system itself. This appears to be reasonable and thus we have decided to extend the other methods to include this compensation. Within this study, we compensated the scanner MTF by a simple division in Fourier space as Jang and Allebach proposed, despite the known issues of the possible degrading influence of noise. In their approach the division is performed in the scanner RGB color space before the calculation of the input/output amplitude ratio.

We have been using compensation by division in numerous experiments and have not encountered any serious problems. Hence we use the division method since it provides reasonable results and can be applied to all different kinds of MTF measurement methods. Thus a direct comparison between the methods is possible. In any case, the scanner should have a good characteristic, since the better the scanner MTF values, the weaker the degrading noise influence. This division should be taken into consideration when interpreting the results.

The scanner also has a color characteristic curve. Figure 3 shows the relation between the devices grayscale values at the output (image file) and the $Y$ values at the input (paper). It can be seen that the relation is not linear.

\section{Slanted Edge Method}

The slanted edge method is often used to measure the MTF of a scanner ${ }^{7}$ or any capture device and can be adapted to measure the MTF of a printing system as proposed by Bang, Kim, and Choi. ${ }^{4}$ A brief introduction to the method is provided in Sec. 3.1, followed by the results in Sec. 3.2. In Sec. 3.3, we investigate the influence of the interpolation method for the generation of the slanted edge, and in Sec. 3.4 we show results from printing edges with reduced contrast. 


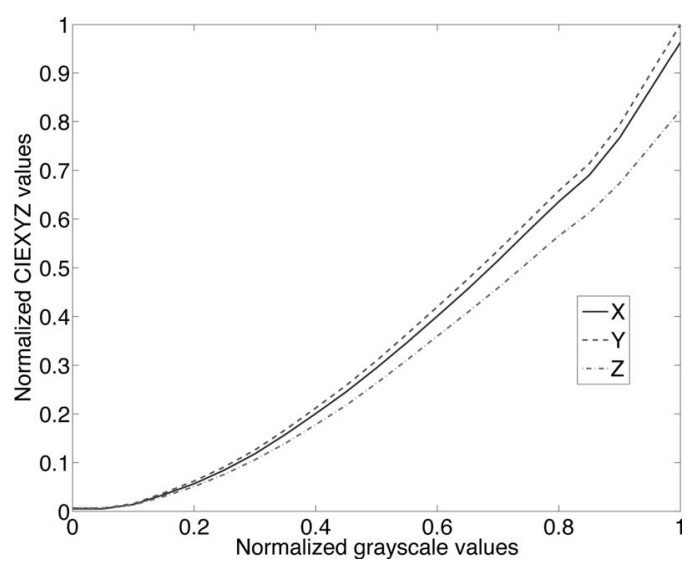

Fig. 3 Scanner grayscale to CIE XYZ conversion.

\subsection{Introduction to the Slanted Edge Method}

An image containing a slanted black square on white background is sent to the printer (see Fig. 4). The printout is scanned and analyzed with the method described in the ISO $16067-1 .^{7}$ We briefly describe this well known method in Fig. 5.

During the analysis, one edge is selected as the region of interest (ROI). Without loss of generality, let us consider the case where the ROI is the left edge of the square. The analysis is visualized in Fig. 5. In each line of the ROI is a transition from black to white-a step function [Fig. 5(a)]. The position of the transition of each step function is estimated and the lines are shifted, so that the transitions are all vertically aligned. Then the average of all the shifted step functions is calculated along this vertical line to reduce the influence of noise [Fig. 5(b)]. The derivative of this mean step function is ideally a Dirac delta function, but in reality it is a peak of a certain width [Fig. 5(c)]. The absolute value of the Fourier transform of this peak is the MTF of the whole system composed of the printing system plus the scanner [Fig. 5(d)].

\subsection{Results}

Figure 6 shows the results of the slanted edge method for the Océ TCS-500. The measure is limited to one edge of

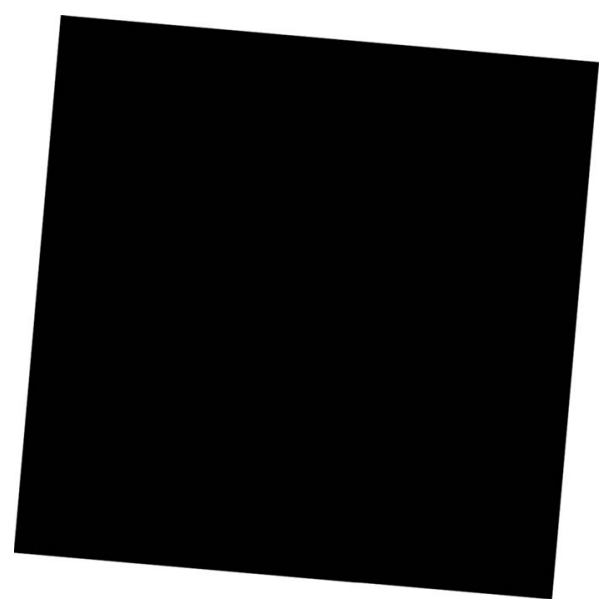

Fig. 4 Slanted square for the slanted edge method.

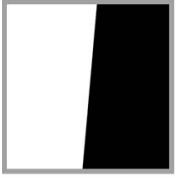

(a)

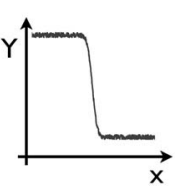

(b)

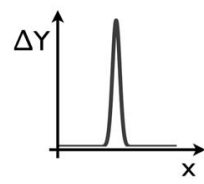

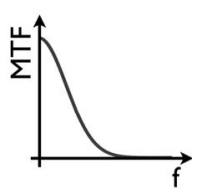

(d)
Fig. 5 Basic principle for the MTF measurement with the slanted edge method: (a) left edge of the square (ROI), (b) average of $Y$ values from shifted lines, (c) derivative with noise suppression, and (d) Fourier transform and normalization resulting in an estimation of the MTF.

full contrast. The MTF is normalized to 1 for the lowest frequency, and decreases when the frequency increases to reach almost zero at the highest frequency 300 cycles/inch.

\subsection{Influence of the Interpolation Method}

A slanted edge can be perfectly described by vectors in a vector image file. However, when sent to a printer it must be rasterized, since printers use a spatial grid of pixels. Different interpolation methods exist to calculate the pixel values along the edge. Hence, when generating the digital image for the slanted edge method, the question of the proper interpolation method arises, since the edges are not parallel to the image grid. To test whether the interpolation method influences the measurements, we generated slanted edges by rotating a square (with horizontal and vertical edges) by an angle of $5 \mathrm{deg}$, using either nearest neighbor or bicubic interpolations. Nearest neighbor and bicubic interpolations were chosen because the first one is very basic and the second one is a more enhanced method. Any influence of the interpolation method should be visible when comparing the results from the two interpolation methods. Figure 6 shows the results from this test on an Océ TCS500: apart from small noise variations, there is no significant difference between the two interpolation methods.

This result is not very surprising, since the very averaging process takes out the difference between the two interpolation methods before sending to the MTF estimation process. But since the interpolation goes beyond the ISO standard for slanted edge measurements, ${ }^{7}$ it was still necessary to investigate this question and to confirm that different interpolation methods do not affect the measurements.

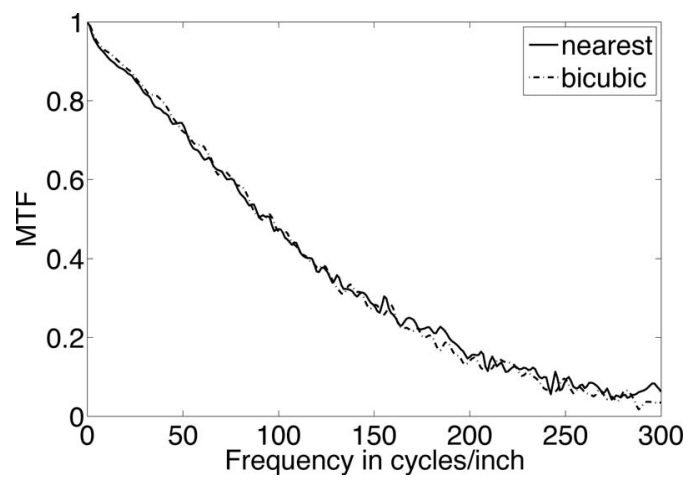

Fig. 6 Result of slanted edge method for a vertical edge created with nearest neighbor or bicubic interpolation printed on an Océ TCS-500. Notice the small noise variation. 


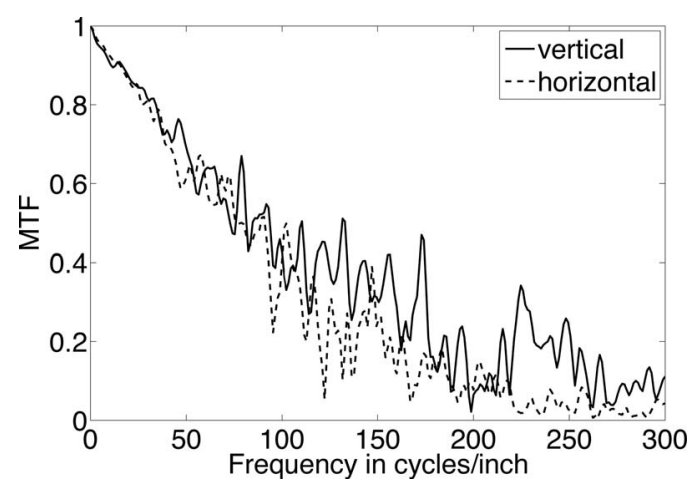

Fig. 7 Result of slanted edge method for a vertical and horizontal edge printed on an Océ TCS-500. Gray level of square: 127 (in an 8 bit image), level of white background: 255 .

\subsection{Influence of the Contrast of the Step}

Since printing systems in general are nonlinear systems, the MTF estimated from a full contrast step might differ from the MTF measured with a step having a reduced contrast. To quantify a possible difference, we have measured the MTF with a step of half contrast: we generated a slanted square with gray level 127 (in an 8-bit image) on white background (level 255). The resulting MTFs for the vertical and the horizontal edge are reproduced in Fig. 7.

We clearly notice that the measurement is affected by much more noise than in the case of full contrast (Fig. 6). Therefore the method does provide a measure of the MTF at full contrast, yet much more effort would be needed to expand the slanted edge method to take into account nonlinearities of printing systems.

\section{Method Proposed in Reference 3}

\subsection{Introduction to the Method}

The method proposed by Hasegawa $e t$ al. ${ }^{3}$ does not measure the MTF directly but through the calculation of the contrast transfer function (CTF). This is achieved by generating a test page with six different pattern types (vertical and horizontal lines, vertical and horizontal stripes, 25 and $50 \%$ duty dot), where each pattern is repeated with different frequencies. An example of one of those patterns ("vertical stripe pattern") is presented in the top part of Fig. 8. To compare the results of this method from the ones of the method proposed by Jang and Allebach (Sec. 5), we have decided to use the same frequencies. Thus we have designed a test page following the design from Hasegawa et al., but with the frequencies set to $\{10,20,30,40,50,60,80,100,150\}$ cycles/inch. In the next step the image is printed and scanned (Fig. 8, middle part). The printer and scanner used are described in Sec. 2. Then the input and output images are matched on top of each other; the difference between the two after the matching is shown in the bottom part of Fig. 8.

One considers for each position $x_{i j}$ its absorbance value $A_{i j}=1-Y_{i j}$ at the output $\left(Y_{i j}\right.$ is the $Y$ value of CIE XYZ at position $x_{i j}$, an intended value at the input $I_{i j} \in\{0$ $=$ white, $1=$ black $\}$, and a local spatial frequency $f_{i j} \in \mathcal{F}$. The means $\bar{A}_{r, f}$ and the variances $V_{r, f}(r \in\{0=$ light, 1 $=$ dark $\}$ ) are calculated for all pixels in the light region $\Omega_{0, f}=\left\{x_{i j} \mid I_{i j}=0, f_{i j}=f\right\}$ and all pixels in the dark region $\Omega_{1, f}=\left\{x_{i j} \mid I_{i j}=1, f_{i j}=f\right\}$, and for each frequency $f \in \mathcal{F}$, respectively:

$$
\begin{aligned}
& \bar{A}_{r, f}=\frac{1}{n_{r, f}} \sum_{x_{i j} \in \Omega_{r, f}} A_{i j}, \\
& V_{r, f}=\frac{1}{n_{r, f}-1} \sum_{x_{i j} \in \Omega_{r, f}}\left(A_{i j}-\bar{A}_{r, f}\right)^{2},
\end{aligned}
$$

where $n_{r, f}=\#\left\{x_{i j} \in \Omega_{r, f}\right\}$ is the cardinality of $\Omega_{r, f}, \quad r$ $\in\{0,1\}, f \in \mathcal{F}$.

Additionally, a solid black (100\% ink) and a paper white (0\% ink) field are analyzed to calculate the averaged maximum value $A_{\text {solidblack }}$ and minimum value $A_{\text {paperwhite. The }}$ $\mathrm{CTF}$ is then defined as:

$\operatorname{CTF}(f)=100 \cdot \frac{\max \left(\bar{A}_{1, f}-\bar{A}_{0, f}, 0\right)}{\left|A_{\text {solidblack }}-A_{\text {paperwhite }}\right|}$,

where the max function sets the CTF to zero if the bright region is darker than the dark region. The MTF can then be calculated with a conversion equation:

$$
\begin{aligned}
\operatorname{MTF}(f)= & \frac{\pi}{4}\left[\operatorname{CTF}(f)+\frac{\operatorname{CTF}(3 f)}{3}-\frac{\operatorname{CTF}(5 f)}{5}+\frac{\operatorname{CTF}(7 f)}{7}\right. \\
& -\ldots] .
\end{aligned}
$$

The variances are used for a significance test that estimates whether the two means are significantly different with respect to their variances and cardinalities. For this test the two quantities

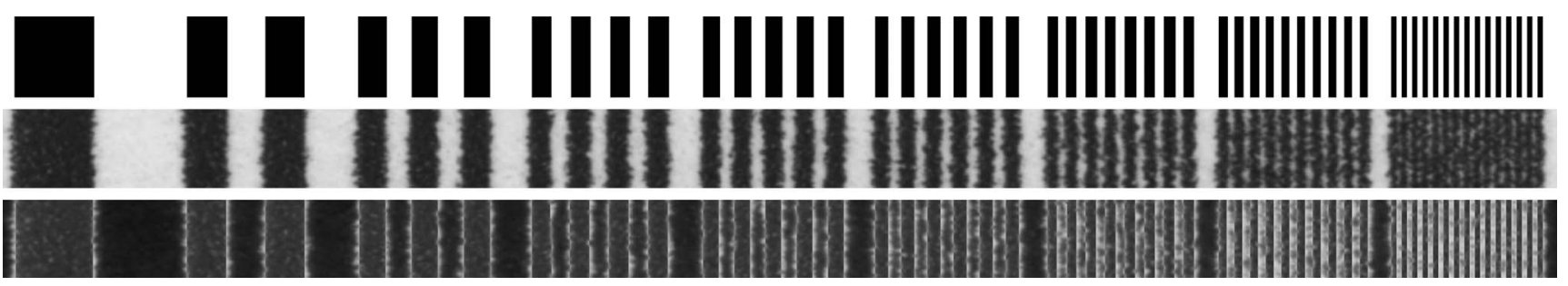

Fig. 8 Enlarged vertical line pattern: input image (top), output image (middle), and difference (bottom). Actual total width $=1$ in. 
$t_{f}=\frac{\bar{A}_{1, f}-\bar{A}_{0, f}}{\sqrt{\left(V_{1, f} / n_{1, f}\right)+\left(V_{0, f} / n_{0, f}\right)}}$,

and

$$
\Phi_{f}^{\star}=\frac{\left(\left(V_{0, f} / n_{0, f}\right)+\left(V_{1, f} / n_{1, f}\right)\right)^{2}}{\left(\left(V_{0, f} / n_{0, f}\right)\right)^{2} / \Phi_{0, f}+\left(V_{1, f} / n_{1, f}\right)^{2} / \Phi_{1, f}},
$$

are calculated, where $\Phi_{r, f}=n_{r, f}-1$ are the degrees of freedom of the light and dark regions, respectively. The statistic $t_{f}$ is related to the student's distribution $\mathcal{I}\left(\Phi_{f}^{\star}, \alpha\right)$ with the significance level $\alpha$ and the estimated degrees of freedom $\Phi_{f}^{\star}$. This leads to the inequality

$\left|\bar{A}_{1, f}-\bar{A}_{0, f}\right| \geqslant z_{(1-\alpha)}^{\Phi_{f}^{\star}} \cdot \sqrt{\frac{V_{1, f}}{n_{1, f}}+\frac{V_{0, f}}{n_{0, f}}}$,

which is fulfilled if and only if the dark and the light regions can be considered as being significantly distinct with a significance level $\alpha$. The value $z_{\alpha}^{\Phi}$ is the $\alpha$-quantile of the student's distribution with $\Phi$ degrees of freedom. In Ref. 3, $\alpha$ has been set to 0.6 .

\subsection{Analysis}

In this method, the use of vector-based images is proposed. The target image sent to the printer is a postscript file (or comparable) with nonpixel data. Many vector graphic documents such as text, technical drawings, or sketches use no intermediate gray (color) levels. In this case it is sufficient to measure the contrast transfer function for black and white (or full color and white) only. Generating a vector graphic test target offers the possibility to generate patterns with any frequency. There is no theoretical limit to the resolution or a constraint that the wavelength has to be a multiple of the pixel size.

The use of rectangular waves instead of sinusoidal waves avoids the introduction of quantization noise observed in Sec. 5.3, yet causes a mixture of different frequencies.

The test of whether the two areas are significantly different or not offers a good add-on to the CTF. This test judges the quality of the measured CTF values. However, we have been able to neither reproduce the results of the significance tests in Ref. 3, nor retrace the choice of the significance level $\alpha$ made in Ref. 3 .

\subsection{Significance Test}

We first propose a simplification of the student distribution for the present usage (Sec. 4.3.1), and then discuss the importance of a quantity in the test (Sec. 4.3.2).

\subsubsection{Simplification of student's distribution}

For a sufficiently high degree of freedom (approximately $>100$ ), student's distribution converges against a normal distribution $\mathcal{N}(0,1)$ (see Ref. 13 and Fig. 9). In our case the degree of freedom (number of scanned pixels) is far above this limit. This simplification is even valid for the case of the maximum frequency, where single black and white pixels alternate in the direction of modulation. Assuming a width of $1 \mathrm{in}$. and a resolution of $600 \mathrm{dpi}$, every single

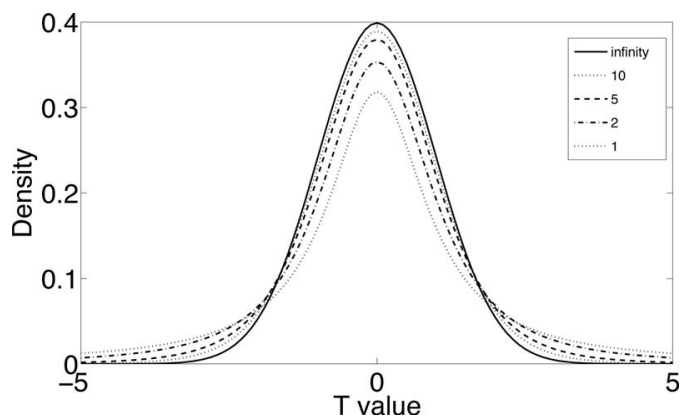

Fig. 9 Student's distributions for different degrees of freedom, converging against the normal distribution.

stripe of the pattern consists already of 600 pixels. In this case, $\Phi^{\star}$ from Eq. (6) does not need to be calculated, and the normal distribution can be used instead.

This is why we only consider the simplified test:

$t=\frac{\bar{A}_{1}-\bar{A}_{0}}{\sqrt{\left(V_{1} / n_{1}\right)+\left(V_{0} / n_{0}\right)}}>z_{1-\alpha}$,

where $t$ is the test statistic and $z_{p}$ is the $p$-quantile of the normal distribution.

\subsubsection{Dependency on the scan resolution}

The significance test depends directly on the cardinalities. If the cardinalities are very high, the variances and means can be considered as well estimated and they will not change. Then the only parameters that change in the calculation of the test variable $t$ are the two cardinalities $n_{0}$ and $n_{1}$.

To demonstrate this dependency, three test patches were generated: one with a very low frequency (3 cycles/in.), another with a higher frequency (50 cycles/in.), and one last with high frequency 300 cycles/in. The three patches were printed at a resolution of $600 \mathrm{dpi}$ and scanned with different resolutions (between 72 and 2400 dpi). Figure 10(a) shows the low-frequency test patch with a dashed rectangle, which indicates the region of interest for the analysis. The stripe on the right, which is outside of the ROI, has been attached to have a continuously periodic signal within the ROI. On the left nothing has to be attached, since it is already white. Figure 10(b) shows the corresponding scan at $600 \mathrm{dpi}$.
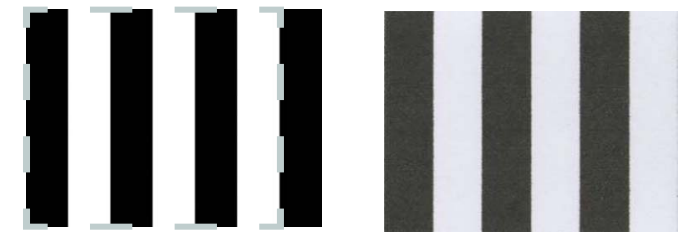
(a) generated test
(b) scan with 600dpi patch

Fig. 10 Low-frequency test patch for the resolution dependency of the significance test. 

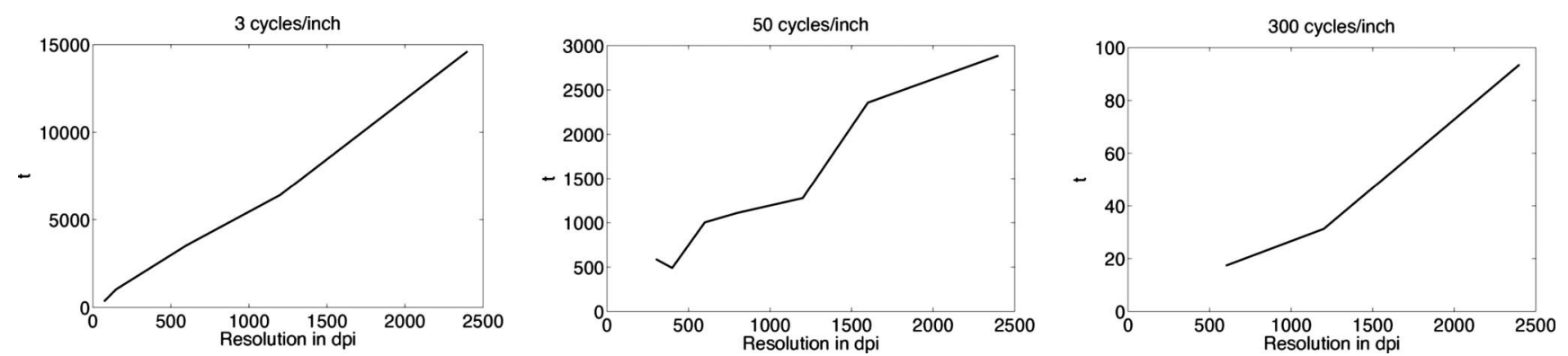

Fig. 11 Statistic $t$ vs the scan resolution for patches with different frequencies; relation is approximately linear.

After scanning the printouts, each with different resolutions (multiples of their frequencies), the images have been analyzed. The variable $t$ versus the scan resolution is shown in Fig. 11. Since the square root of the number of drawings $\sqrt{n}$ is proportional to the scan resolution, the statistic should be proportional to the scan resolution too-this is clearly visible.

From Fig. 11 and inequality 9, it is also evident that there is indeed a cutoff frequency above which the inequation is no longer fulfilled. Figure 11 clearly shows that the test statistic $t$ falls with rising modulation frequency and thus becomes smaller than the limit $z_{1-\alpha}$.

Hence a scan resolution much higher than the print resolution is not justifiable, as this will not result in more information about the printout. Similarly, a scan resolution much lower than the print resolution is not of interest, since it would cause a loss of information, especially at the borders between light and dark areas. It is therefore advisable to choose a scan resolution in the same order as the printer resolution, e.g., twice the printer resolution as in Jang and Allebach. It is important to understand that the result of the hypothesis test depends on the scan resolution.

\subsection{Conversion Equation: Contrast Transfer Function to Modulation Transfer Function}

Seen apart from the inconvenient resolution dependency of the hypothesis test, one can calculate the values of the CTF and neglect their significance. For each of the six pattern types one obtains a CTF. Figure 12 shows the CTF for the horizontal and vertical stripe patterns on the left, and in the middle the MTF calculated with the proposed conversion equation. The two stripe patterns have been chosen because their structure is closer to Jang and Allebach's patches than the structure of the other four patterns. The MTF from the other four patterns is shown in Fig. 12 on the right. To calculate the MTF with Eq. (5) for the frequency $f$, one needs the CTF at odd multiples of $f$. In our conversion we interpolate intermediate CTF values linearly and extrapolate values for frequencies above 150 cycles/inch but set them to zero when they become negative.

\subsection{Results}

The MTFs obtained with the method from Hasegawa et al. differ significantly from each other and it is not obvious which one to choose to get a good estimation of the real MTF. Like the MTF obtained from Jang and Allebach's method, this method also estimates a better transfer for modulations in the vertical direction (note that horizontal stripes correspond to vertical modulation and vice versa). So there is a coherency between the two methods.

\section{Method Proposed by Jang and Allebach}

This section begins with an introduction to the method, Sec. 5.1 followed by a short summary of the linearization step proposed in Lindner et al., ${ }^{5}$ Sec. 5.2. The influence of the amplitude of the sinusoidal waves is evaluated in Sec. 5.3. A newly modified printer MTF measurement method is proposed: it is simpler to implement and provides more reliable results.

\subsection{Introduction to the Method}

Jang and Allebach's method ${ }^{4}$ consists of printing sinusoidal patches and comparing their amplitudes with constant tone patches. The constant tone patches have uniform values that are also the maximum, the mean, and the minimum values of the sinusoidal patches.
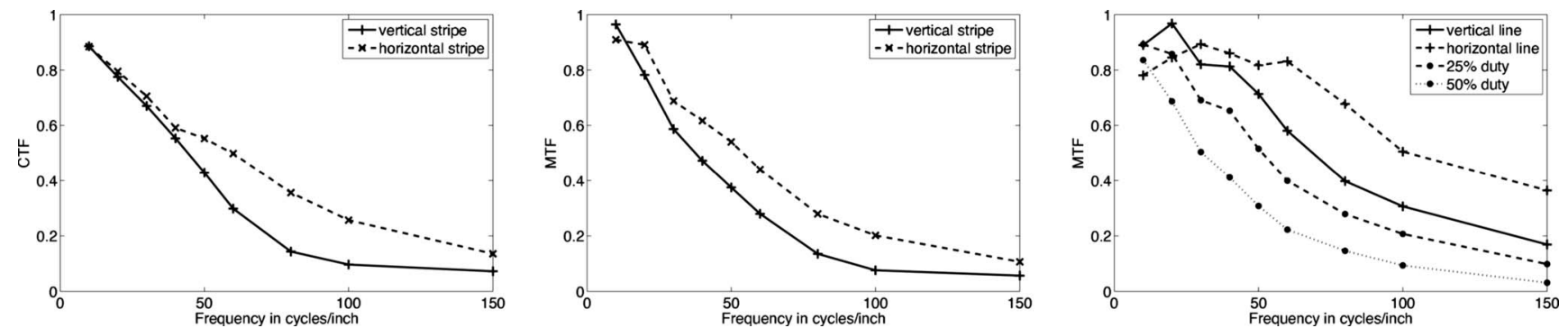

Fig. 12 Left and middle: CTF and MTF for the horizontal and vertical stripe pattern. Right: MTFs for the other four test patterns calculated from their corresponding CTFs. 


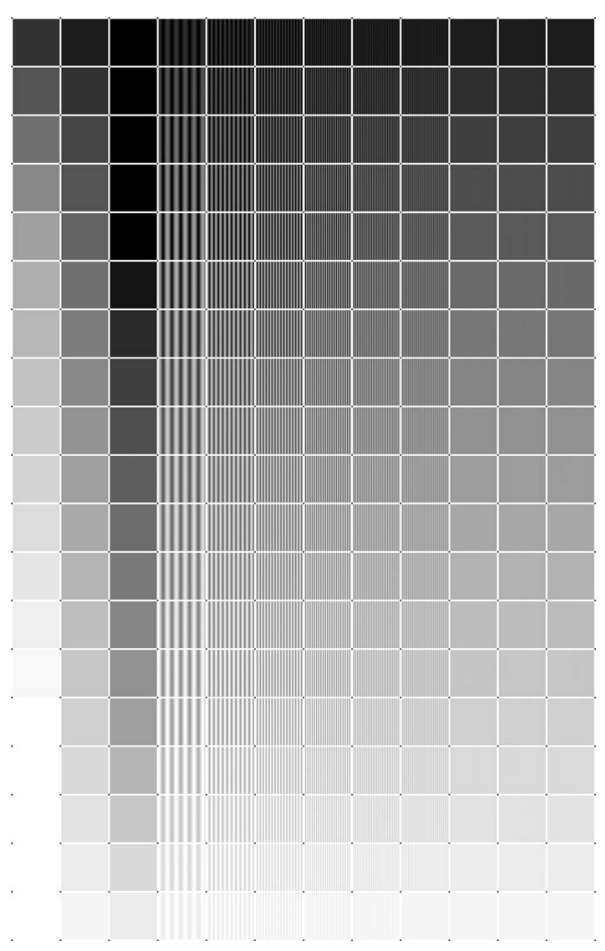

Fig. 13 Jang and Allebach's test image.

One row of the test image consists of these three constant tone patches and the nine corresponding sinusoidal patches with frequencies $f$ $\in\{1020304050608080100150\}$ cycles/inch. The whole test image consist of 19 rows to measure the MTF with different biases. An example of such a test image is illustrated in Fig. 13.

The analysis is depicted in Fig. 14. It can shortly be described as follows.

1. Within one row, the three constant tone patches are processed first. For each patch, its mean is calculated in the CIE XYZ space and converted into CIELAB values.

2. For each of the nine sinusoidal patches from the same row, the modulation signal is extracted by averaging the measured tristimulus values perpendicularly to the direction of modulation. The averaged values are converted to CIELAB values and projected on the line that connects the lower and upper mean values corresponding to the constant min and max tone patches, respectively.

3. Then, for all the points projected on the line, the $\Delta E_{a b}^{*}$ distance to the lower mean value of the constant min patch is calculated. The result is a vector of $\Delta E_{a b}^{*}$ scalar values, which is Fourier transformed. The amplitude of the main frequency of the patch is then extracted.

4. The amplitude is compared with the $\Delta E_{a b}^{*}$ distance between the constant min and max tone patches and it is usually smaller. Since the scanner is not compensated at this point, their ratio is not yet the printer MTF. It is the MTF of the system composed by both the printing system and the scanner.

5. For the scanner compensation, we use the scanner MTF, which has been separately measured with specific engraved patterns on a physical chart. ${ }^{7}$ We then estimate how much the scanner attenuates a signal that oscillates between the min and max constant tone patches. This scanner ratio should be between 1 and the ratio calculated in the previous step. Dividing the first calculated combined printer and scanner ratio by the prior scanner ratio provides the estimated compensated printer MTF.

\subsection{Analysis}

This method offers the possibility to measure the MTF for different bias levels, orientations (e.g., horizontal and vertical), and colors. With these parameters, there are many different varieties of measurements possible that are a-priori interesting. Additionally, it includes a method for the compensation of the scanner MTF to calculate the MTF of the printing system only.

The possibility of color measurements would be relevant to obtain a complete printer model. However, we have not made use of that in this work: since the human visual system (HVS) is more sensitive to luminance high-frequency content than chrominance high-frequency content, our interest is mainly to evaluate the luminance-based MTF, not the chrominance-based MTF (see also Sec. 8). This method does not take into account the halftoning and noise introduced by the printing system. By taking care of them, one could gain more reliable measured values. At first, we obtained MTF values significantly greater than 1 . Since this is principally impossible, it showed a need to further investigate. In the following Sec. 5.3, we discuss the noise and its influence on the measured MTF values.

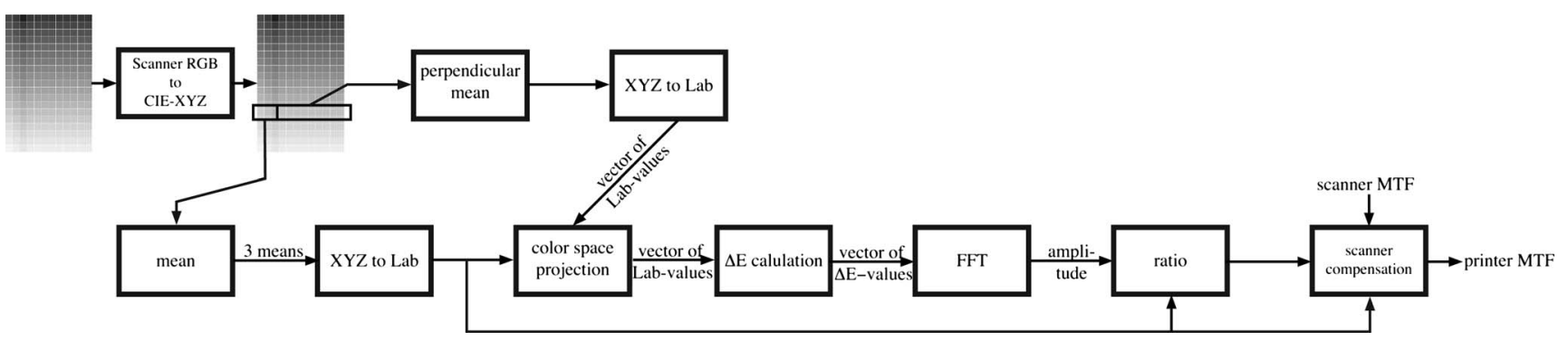

Fig. 14 Flowchart of the method proposed by Jang and Allebach. 

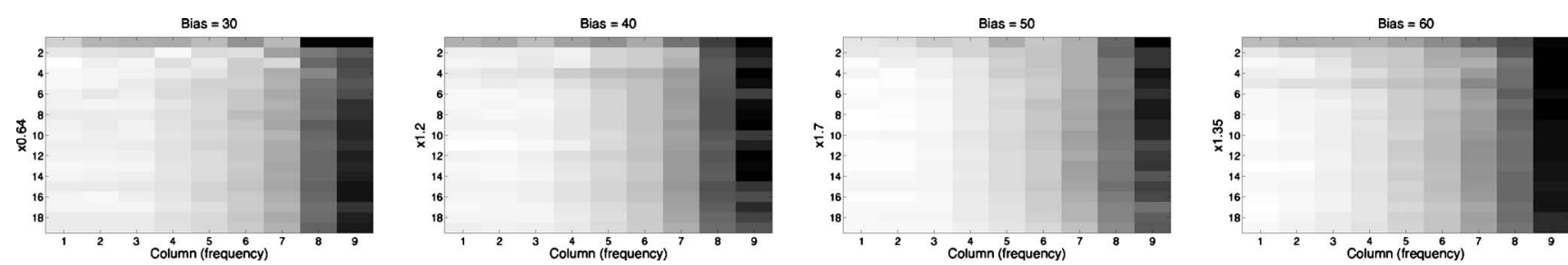

Fig. 15 Amplitude dependency of the MTF for different mean values. The MTF value is shown as a gray level (the brighter the rectangle the higher the corresponding MTF value).

\subsection{Modulation Transfer Function Values Greater than 1-Amplitude Dependency}

In our first attempt to reproduce Jang and Allebach's experiments, several measures of the MTF were greater than 1. Theoretically this should not happen, since the processing workflow of our printing process contains neither any contrast enhancement nor any similar processing. This problem turned out to be related only to the physics of the printer, not with the concept of the method itself.

The exceeding MTF values showed a random behavior, and no systematic rule could be concluded from the measurements of several printouts. This suggested that the problem was due to the presence of strong noise in the patches. The easiest way to gain better results was to use higher amplitudes for the sinusoidally modulated patches. Thus the influence of the amplitude on the measurement has been analyzed in two steps. First the influence has been quantified and qualified (Sec. 5.3.1), and second the test page from Jang and Allebach has been modified on the basis of this new knowledge (Sec. 5.3.2).

\subsubsection{Finding an optimal amplitude}

To investigate the influence of the amplitude $\Delta Y=\left(Y_{\max }\right.$ $\left.-Y_{\min }\right) / 2$ on the measurement, a special test page has been designed. This test page does not contain sinusoidal patches with different bias levels and constant amplitudes as proposed in Ref. 4, but the whole test page has the same bias level $Y_{\text {bias }}$ and the rows differ in their amplitudes. The amplitude in the $n$ 'th row is $n / 19 \cdot \min \left(Y_{\text {bias }}-Y_{\text {low }}, Y_{\text {high }}\right.$ $\left.-Y_{\text {bias }}\right), n \in[1,19]$. That means that the amplitudes grow linearly along the columns. The maximum amplitude is chosen such that the sinusoidal wave then reaches either the upper or lower border of the printable interval $\left[Y_{\text {low }}, Y_{\text {high }}\right]$. For example, for a bias level of $Y_{\text {bias }}=30$ and a lower border of $Y_{\text {low }}=17.8$, the amplitudes are $n \cdot 0.64$, as indicated at the ordinate at the very left of Fig. 15.

The images in Fig. 15 illustrate the four different bias levels the MTF values as a grayscale image.

These images show that for very low amplitudes (top rows), the measured MTF values become significantly noisy, and for larger amplitudes the MTF values are more constant.

To investigate this behavior, the variances in the first column of the four MTFs with different biases have been plotted (Fig. 16). The images in Fig. 15 clearly show that the biggest changes take place for the lower amplitudes. Therefore it is interesting to calculate the variance of the values from the $n$ 'th to the last row. For small $n$, the noisy values from the first rows are considered and induce a high variance; for high $n$ only the stable MTF values are left over and the variance is smaller. The curves are from the first column of each of the four MTFs. On the abscissa there is the $Y$ value from which the MTF values have been considered for the variance.

Figure 16 shows that from a certain amplitude on, the variances of the measured MTF values are quite low compared to the variance of all the measured MTF values. This amplitude is approximately $\Delta Y \approx 5$, and it is advisable to use sinusoidal modulations with higher amplitudes. Further on, the low variance of MTF values above the limit amplitude allows us to elevate the amplitude without strongly affecting the measured MTF values.

\subsubsection{Modifying Jang and Allebach's test image}

In the paper from Jang and Allebach, ${ }^{4}$ the amplitude $\Delta Y$ is restricted to not having to crop the sinusoidal patches at the limits of the printable interval $[0 \ldots 255]$ in our case, this amplitude corresponds to approximatively 3 on the $Y$ axis.

In our new test image, the bias levels $Y_{i}, i \in[1,19]$ are chosen such that $\left\{Y_{\text {low }}, Y_{1}, \ldots, Y_{19}, Y_{\text {high }}\right\}$ are equidistantly distributed. The amplitude is constant where possible and greater than 5. Exceeding $Y$ values are avoided by reducing the amplitude for those bias levels where necessary. The amplitudes are thus constant in the middle part of the test image and fall down at the most upper and lowest rows of the test image (darkest and lightest rows, respectively).

Even though experiments (Fig. 17) showed that for sufficient high values, the amplitude does not affect the MTF measurement, we prefer to vary it as less as possible within one measurement. Thus, on the one hand the amplitude should not be chosen too high to avoid lowerings in too many rows due to the borders of the printable $Y$ range. On the other hand, the amplitude should be chosen sufficiently high to reduce noise in the measurements. Based on our experiments we chose an amplitude of $\Delta Y=5$. It has to be

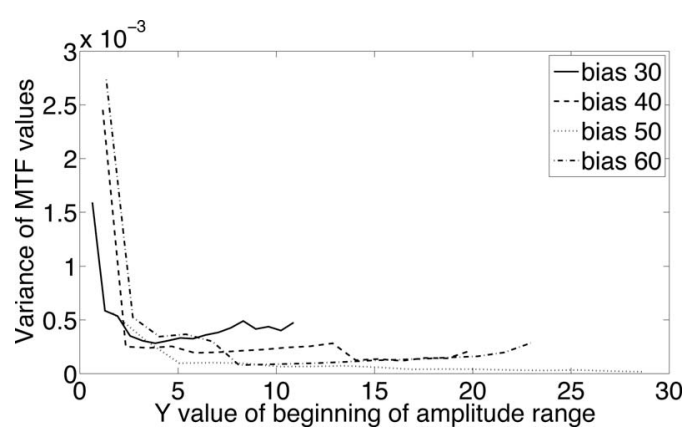

Fig. 16 Variances of MTF values vs different amplitude ranges; curves from four MTFs with different bias levels. 

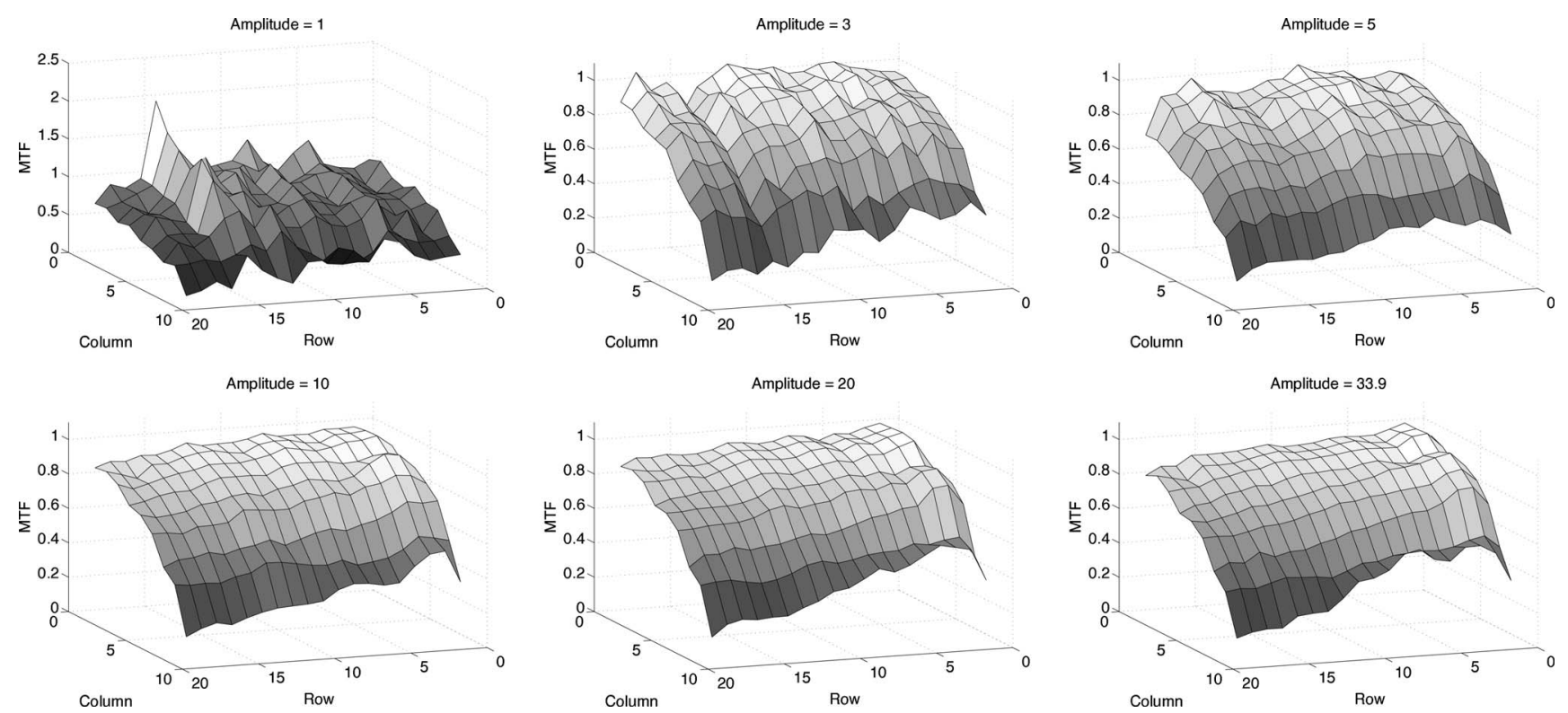

Fig. 17 MTFs measured from new test images with different amplitudes; in each subfigure the row shows increasing bias level and the column shows increasing frequency.

remarked that this value is most likely different for different printing systems-a (nonrecurring) preliminary investigation before measuring the MTF of a printing system seems unavoidable.

Furthermore, to avoid errors due to nonlinear conversions from CIE XYZ to CIELAB (for an extended discussion, see Ref. 14), we propose to generate the test page and do the analysis on the $Y$ axis of CIE XYZ. In this case the input amplitude can be estimated as the distance between the max and min constant tone patches, since the signal is not systematically distorted. This approach has three major improvements. First, the analysis is much simpler, since it needs neither the projections nor the $\Delta E_{a b}^{*}$ measures in CIELAB space (the method initially proposed used those calculations $\left.{ }^{4}\right)$. Secondly, the ratio $A_{\text {out,Fourier }}(f) / A_{\text {in,space }}$ can be used without corrections. Thirdly, the measure provides a physical estimation of the printing system MTF, since it uses the $Y$ value for the analysis instead of the perceptual $\Delta E_{a b}^{*}$ distance.

For the MTF measurement, only the minimum and maximum constant tone patches are needed and the middle constant tone patch can be disregarded. Yet Jang and Allebach included it in the design of their test page, and therefore we left it on our test page too. This patch can be used to check whether the linearization of the printing system to the $Y$ axis is correct. If for instance the mean $Y$ value of the middle constant tone patch is not equal to the means of each sinusoidally modulated patch (in the same row of the test page), the linearization has not been done properly.

\subsection{Results}

\subsubsection{Modulation transfer function of the Océ Colorwave 600 inkjet printer on uncoated paper}

A resulting MTF from the new measurement method, for the Océ Colorwave 600 inkjet printer on uncoated paper, is shown in Fig. 18. Unlike in the article from Jang and
Allebach, ${ }^{4}$ in this study we limit our investigation to grayscale (using black ink) and thus do not consider color (e.g., cyan, magenta, or yellow) inks. The procedure (linearization followed by printer MTF measurement) can be extended to any other ink. The yellow ink is a special case: errors can occur since the $Y$ values of yellow ink are usually very high even for full ink coverage, and thus the generation of large amplitude sinusoidal patches is not possible.

\subsubsection{Modulation transfer function of other printing systems}

Figures 19 and 20 show the measured horizontal MTFs of the Océ TDS 700 and HP 5500 inkjet printers on uncoated paper. The shape of these two measurements interestingly shows the variety of MTF, depending on characteristics of the printing systems.

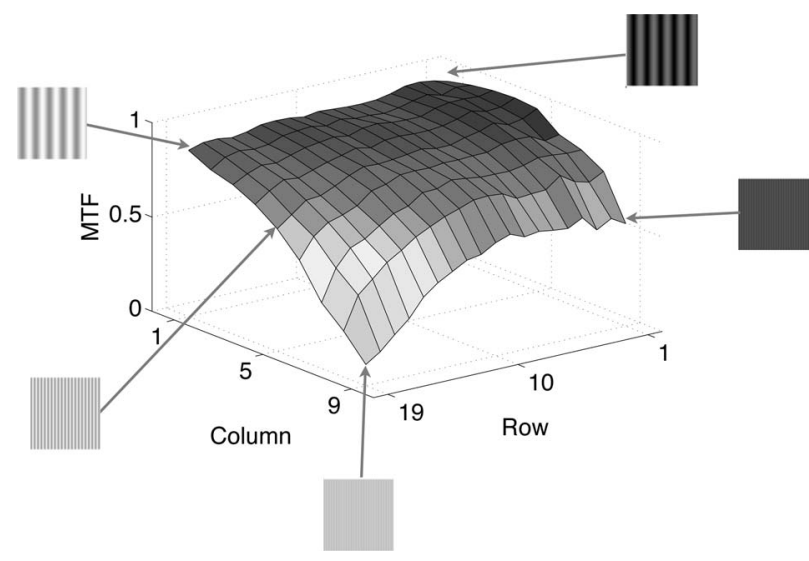

Fig. 18 Horizontal MTF of the Océ Colorwave 600 inkjet printer on uncoated paper obtained with our new measurement method based on Ref. 4. Example patches and arrows have been added for some MTF values for illustration purposes. 


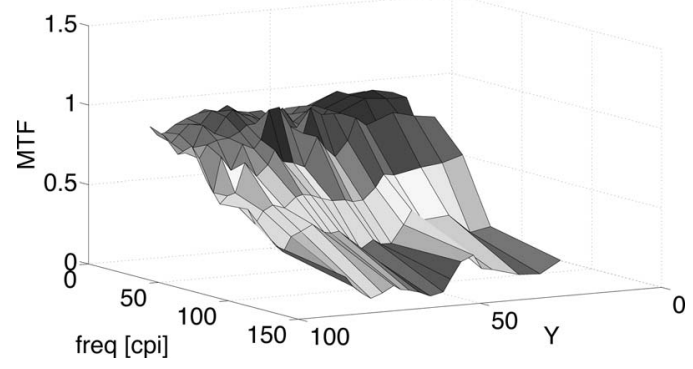

Fig. 19 Horizontal MTF of the Océ TDS 700 inkjet printer on uncoated paper.

\subsection{Modulation Transfer Function in Horizontal and Vertical Directions}

We compare the MTF of two test images with spatial modulation in the horizontal and vertical directions with the amplitude $\Delta Y=5$. Figure 21 shows the vertical [Fig. 21(a)] and the horizontal MTF [Fig. 21(b)], and their ratio [Fig. 21(c)]. Note that in Fig. 21(c), the axes of the plot have been rotated counterclockwise by $90 \mathrm{deg}$ to avoid occlusions.

As one can see, the MTF values in both directions are very close to 1 for the lowest frequency and they are not exceeding 1. Furthermore, the distinction between the two directions emerges as being important, as can be seen in Fig. 21(c): for high frequencies (columns of high rank), the reproduction of sine waves is significantly better in the vertical than in the horizontal direction.

Finally there is a falloff in the light region (high row number), which is probably due to the halftoning process. The brighter a patch, the lower the droplet density and the higher the mean distance between them. When this mean distance is in the order of magnitude of the sinusoidal wavelength, it becomes very difficult to reproduce the frequency, as shown in Fig. 22. In high-end printing systems, the use of gray ink allows us to avoid such issue.

\subsection{Modulation Transfer Function in Intermediate Angle Directions}

Since natural images contain not only horizontal and vertical modulations but a mixture of modulations in any possible direction, the MTF should be known for intermediate angles to fully describe the behavior of a printer. As previ-

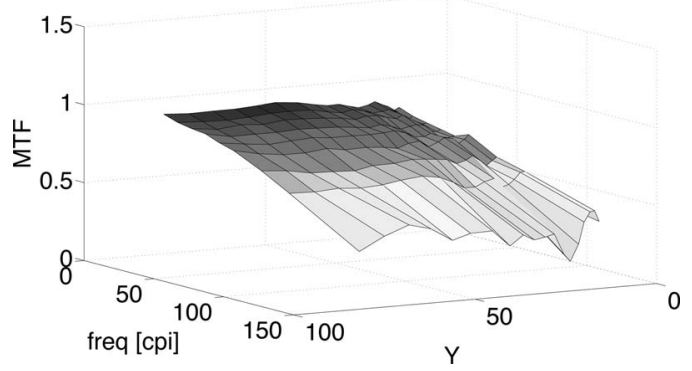

Fig. 20 Horizontal MTF of the HP 5500 inkjet printer on uncoated paper.

ously mentioned, the printer principal directions are the horizontal and vertical. For reasons of simplicity it would be beneficial to interpolate the MTF for intermediate angles from the two MTFs in the principal directions.

To test whether this is possible, we numerically rotate the test page with the amplitude $\Delta Y=5$ by 45 deg (clockwise) and $-45 \mathrm{deg}$ (counterclockwise), and print those two versions. Then we scan and analyze them as usual.

The first important point to notice is that the two MTFs (45 and $-45 \mathrm{deg}$ ) are very similar. We calculated their ratio patch by patch. The $(9 \times 19=) 171$ ratios have a mean of 0.99 and a standard deviation of 0.005 , which means that they can be considered (seen apart from noise) as identical. Thus the direction of rotation is not as important as the absolute value. The next analysis can be undertaken with one of the rotated images. In the following we have selected the clockwise rotated image.

We then calculate the ratio of the 45-deg MTF to both the horizontal and vertical MTFs; these two ratios are illustrated in Fig. 23.

As one can see, the values of the clockwise MTF lie between the vertical and horizontal MTF values. This is interesting because it could be possible to interpolate the MTF for an angle $\theta$ as the weighted sum of the horizontal and vertical MTFs:

$\mathrm{MTF}_{|\theta|}=f(|\theta|) \cdot \mathrm{MTF}_{\mathrm{hor}}+(1-f(|\theta|)) \cdot \mathrm{MTF}_{\mathrm{ver}}$,

where $f(|\theta|):[0,90 \operatorname{deg}] \mapsto[0,1]$.

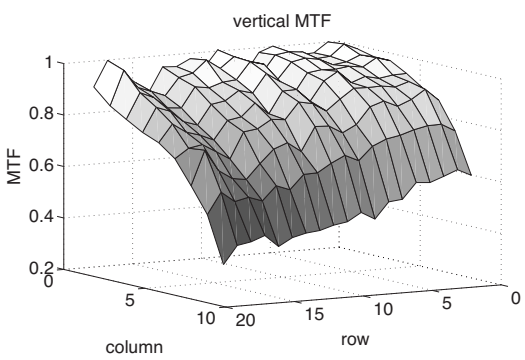

(a)

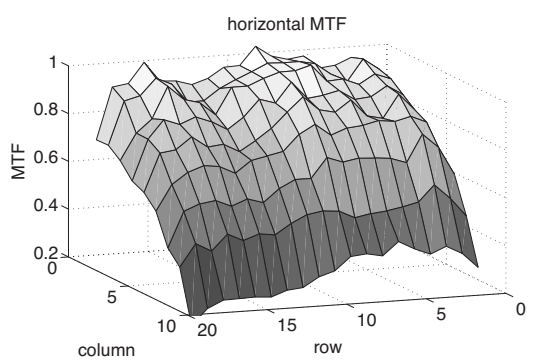

(b)

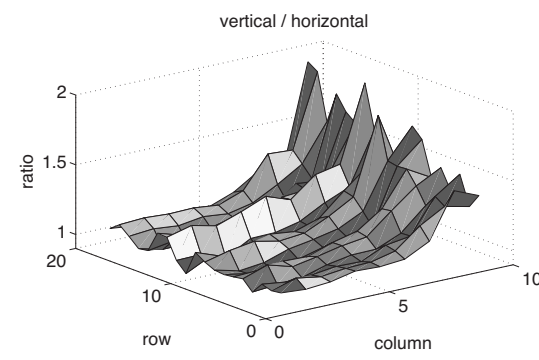

(c)

Fig. 21 Comparison of vertical and horizontal MTF and their ratio. Rows show increasing bias level, columns show increasing frequency. In (c) the column and row axis have been oriented differently than in previous figures for better visualization. 

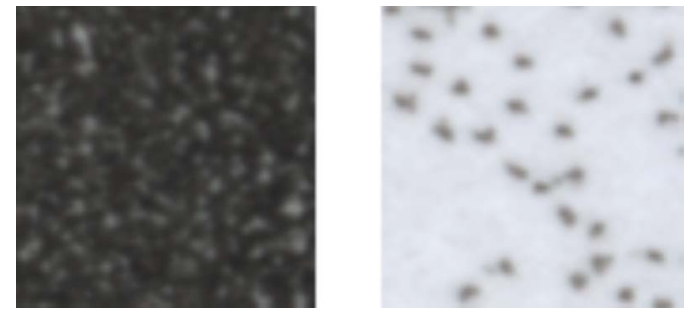

Fig. 22 Darkest (row 1) and brightest (row 19) patches of the column (rank 9$)$ with the highest frequency $(80 \times 80$ pixels at $1200 \mathrm{dpi})$.

$f(\theta)$ is most likely a monotone function and-due to the symmetry-has to be defined only for angles in the interval $[0,90 \mathrm{deg}]$ instead of $[-90,90 \mathrm{deg}]$.

\subsection{Discussion}

The benefits from generating and analyzing modulations along the CIE XYZ $Y$ axis should be highlighted. The modulation could be generated using other color spaces, at least as long as the same other space is used throughout the whole process to avoid nonlinear distorsions as discussed in Ref. 14 (Fig. 3 shows the nonlinear characteristic curve of the scanner). The CIE XYZ $Y$ axis has the advantage to be a device-independent quantity, which enables comparisons among different measures. This quantity is linearly related to the physical quantity of reflection of the printed medium and the amount of ink on the medium. Additionally, a workflow based on the $Y$ axis can be used to measure the MTF for a signal modulating along any color axis. The usage of device-dependent RGB values from the measuring scanner would result in evaluations of the MTF biased by the nonlinearities in the scanner response. It would also complicate comparisons between different devices.

Noise is another topic of interest. In the course of this study we have considered the noise in the method proposed by Jang and Allebach. ${ }^{4}$ We designed a special test image where the bias level is constant and the amplitude is different in each row. The results show clearly that low amplitudes have two disadvantages: measurements are noisy and tend to be significantly smaller than 1 , even for low frequencies.

Furthermore, it was found that the MTF values depend far less on the amplitude when the amplitude is above a certain threshold $(\Delta Y \approx 5$ in our experiment). We have thus introduced a modification to Jang and Allebach's test image by setting the amplitude to this optimal larger value. Fi-

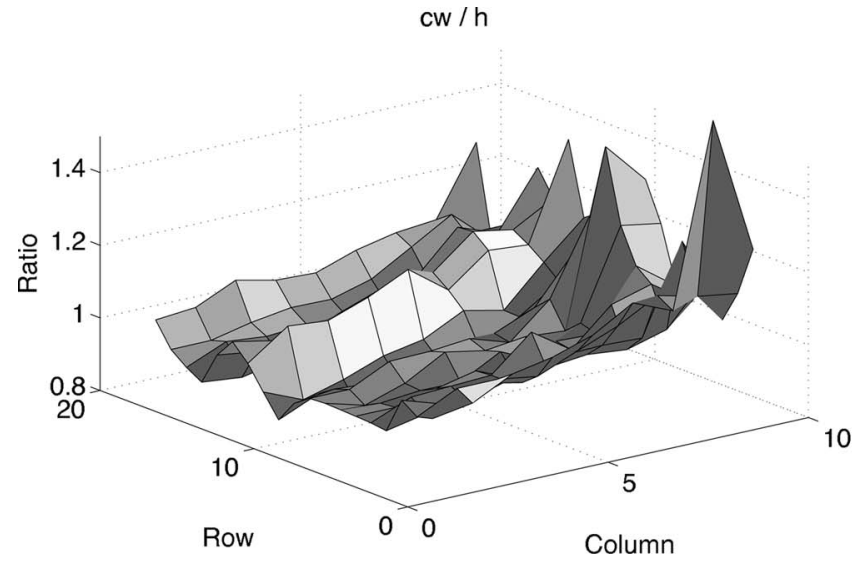

(a)

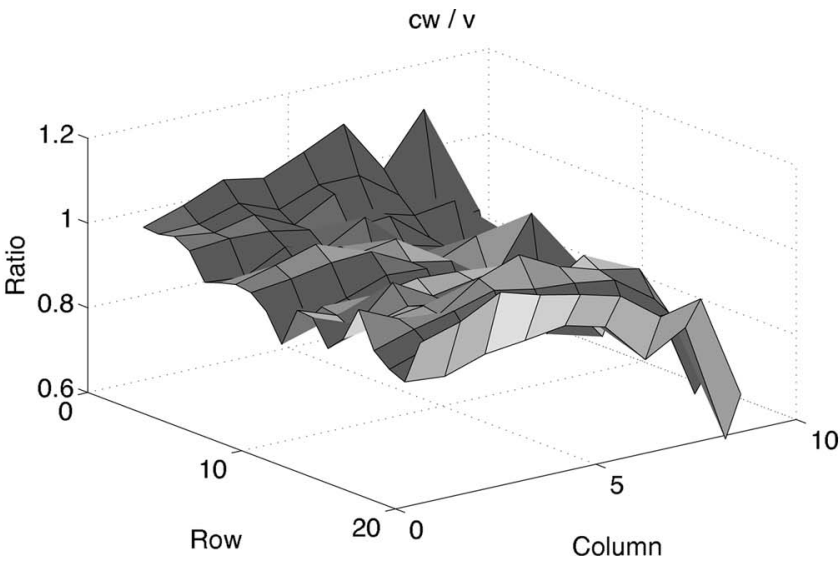

(b)

Fig. 23 Ratios of the 45-deg MTF to the horizontal (a) and vertical (b) MTF. Rows show increasing bias level, columns show increasing frequency. In these two figures, the column and row axis have been oriented differently than in previous figures for better visualization.
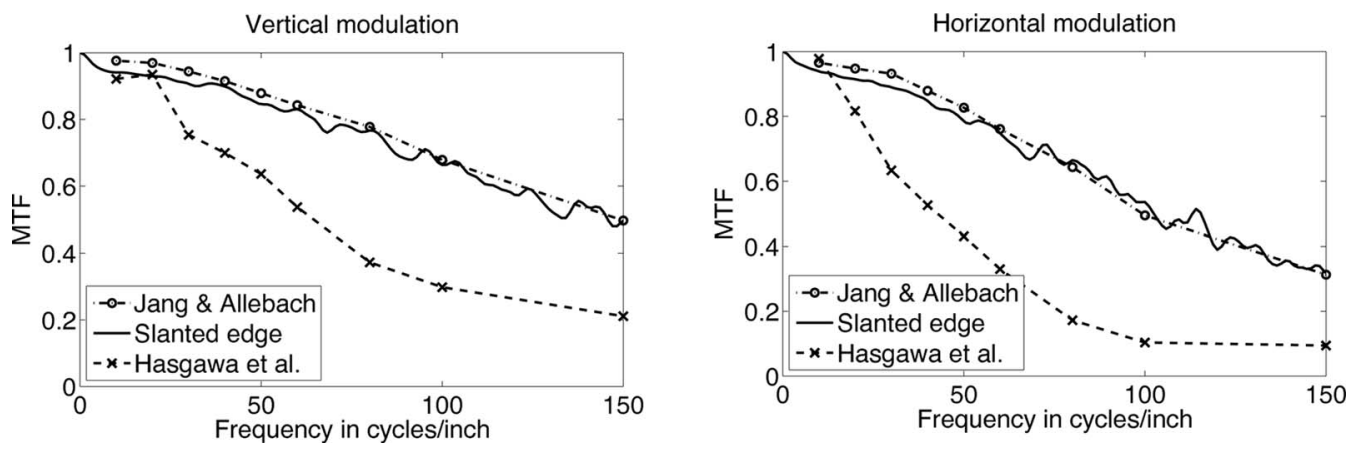

Fig. 24 MTF measures from all three methods (Jang and Allebach, ${ }^{4}$ slanted edge ${ }^{7,9}$ and Hasegawa et al. ${ }^{3,5}$ ) for direct comparison. Values from Hasegawa et al. and the slanted edge method have been compensated by simple division. Jang and Allebach's values are from the darkest gray rows (the average of the first three rows of the test page). 
nally, we showed that with our experimental setup, the MTFs from test pages that had been rotated (45 and $-45 \mathrm{deg}$ ) before printing have the same values, and that these values lie halfway between the horizontal and vertical MTF values. Hence, it is most likely possible to interpolate the MTF for an intermediate angle from the horizontal and vertical MTF.

\section{Comparison of the Results and Discussion}

The MTF values from the three methods are plotted in Fig. 24 for direct comparison. It is necessary to add that the MTF values from Jang and Allebach's method are different for different bias values. The ones shown in Fig. 24 are from the darkest rows of the test page (the average of the first three rows of the test page). The MTF values from lighter patches are slightly lower (as can be seen in Fig. 18).

The results from the slanted edge method and Jang and Allebach's method are very close to each other, while the resulting curve from Hasegawa et al.'s method is significantly lower. This is not very surprising, since Jang and Allebach's and the slanted edge method are conceptually related. They both directly measure the frequency response of the printing system: the slanted edge method by a Fourier transform of the derivative of a step function (Fig. 5) and Jang and Allebach's method by estimating the attenuation for a fixed set of sample frequencies (Fig. 14). Instead of that, the method from Hasegawa et al. measures first a CTF, which is then converted to an MTF with an infinite series [Eq. (5)].

The method from Hasegawa et al. ${ }^{3}$ is sensitive to phase shifts. For example if the printed rectangular pattern is shifted by a quarter of the wavelength, the measured CTF is equal to zero. The exact measurement of phase shifts depends strongly on the quality of the cross-marks used for positioning in the analysis. Since the cross-marks are printed as well, they can also be affected by phase shifts, which can falsify the result. The method from Hasegawa et al. has another drawback: it measures first the CTF, which has then to be converted to the MTF. The conversion can be error prone, since it depends on an infinite series. This also causes the MTF values for low frequencies to be affected by the noise from high-frequency CTF values. Additionally, we do not presently dispose of a proof of the meaningfulness of the conversion. Even though theoretically proven, ${ }^{15}$ it is not necessarily applicable to printer MTF measurements.

Conceptually, the method from Jang and Allebach and the slanted edge method are closer related to the definition of an MTF (a ratio of amplitudes), since they actually measure this ratio in the frequency domain. Hasegawa et al.'s method performs area comparisons, which are, by construction, different from amplitude ratios for specific frequencies. Only the detour with the conversion equation gives an estimate of the MTF. Additionally, Hasegawa et al. do not explain how to exploit the different CTF/MTF curves obtained for the different test patterns (Fig. 12), or whether it could be useful to design even more test patterns. Thus, to determine the resolution of printing systems or for quality tests, the CTF can be used and it is not necessary to calculate an MTF. But since our goal is then to use the measurements for further image processing based on the printer
MTF, ${ }^{16}$ we would have to use the conversion. The uncertainty of the conversion makes Hasegawa et al.'s method less favorable for our needs. Thus, the remaining question is whether to use the method from Jang and Allebach or the slanted edge method.

The slanted edge method ${ }^{7,9}$ is very simple to implement and the test image (Fig. 4) is both very easy to create and handle. In addition, the method has already become an ISO standard for scanner MTF measurements, ${ }^{\top}$ and thus there is already experience in using it. The drawback of the slanted edge method is that it does not take into account the bias dependency of the MTF. An attempt to modify the slanted edge method by printing a step with reduced contrast (e.g., a step from 0 to $50 \%$ ink coverage) has led to results strongly affected by noise (see Fig. 7). Therefore, more effort would be needed to expand this method to measure bias dependent MTF values.

The method from Jang and Allebach shows that the attenuation of a printed sinusoidal signal depends indeed on its bias value. Since it already takes into account this dependency when the other two methods do not, Jang and Allebach's method is more related to our needs. It provides a more exhaustive measurement of the printing system. The importance of the bias dependency becomes clearly evident in the next sections, in which we propose an MTF compensation based on the measurements presented here.

The propositions in Ref. 5 and Sec. 5 help to make the method from Jang and Allebach ${ }^{4}$ more stable and less errorprone. The linearization step and creation of the sinusoidal patches on the $Y$ axis respect the fact that a printer MTF is a physical measure and assure unaltered sine waves (seen apart from quantization errors). The decision to analyze the data from the $Y$ channel instead of the approach in CIELAB bypasses the introduction of errors by nonlinear transformations.

But the method from Jang and Allebach also has its drawbacks. It is rather complicated to implement and handle. For example, it is important to carefully place the printed test image on the scanner, since even small angles between the direction of modulation and the scanner pixel grid may cause unexpected attenuation when calculating the mean of the sinusoidal patch perpendicular to the direction of modulation. Additionally, we had not yet tested how the method (with the proposed modifications) performs with different inks or with different printing and scanning resolutions. Mainly for the yellow ink, it can be a disadvantage to not use full contrast test images as it is done with the method from Hasegawa et al. or the slanted edge method, since yellow ink has a lower dynamic range on the $Y$ channel. This could cause noise and have a serious impact on the measurement.

The purpose and context influence the choice of the measuring method. If it is not intended to generate test pages adapted to the printing system, one could use the slanted edge method or the method from Hasegawa et al. Those two methods may also be favored if the device is characterized for images with step-like content, since the test images are a more realistic simulation. If an MTF is needed, the method from Jang and Allebach and the slanted edge method should be given preference, since they measure the MTF directly. If a more precise and complete mea- 


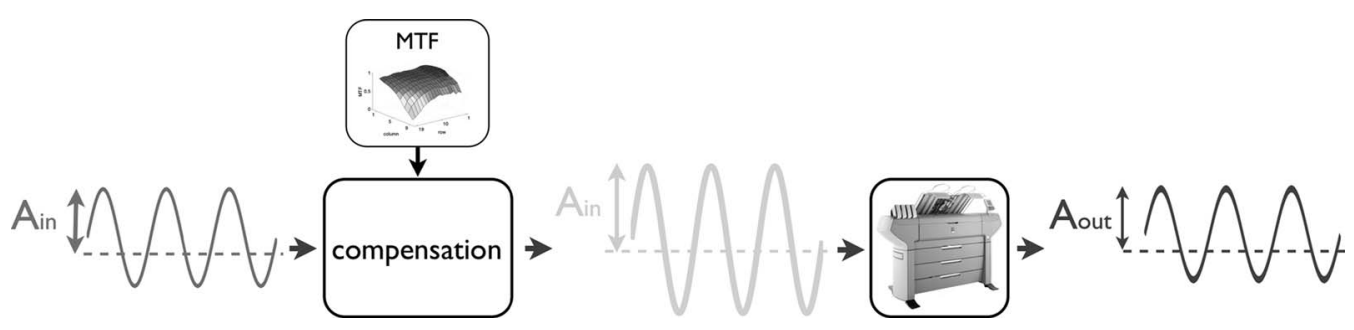

Fig. 25 Principle of the MTF compensation: the amplitude $A_{\text {in }}$ is compensated (preamplified) by compensation (division by the MTF in the Fourier domain) before printing, resulting in a printed output amplitude $A_{\text {out }}$ close to the input $A_{\text {in }}$.

sure is needed for images with different local gray levels, the method from Jang and Allebach should be used, since it takes into account the bias dependency.

Overall, the method from Jang and Allebach (together with our improvements) matches very well the requirements we formulated in Sec. 2.2: averaging the patches perpendicularly to the direction of modulation helps making a quasicontinuous signal from the dot grid, and the consistent choice of the $Y$ axis throughout the procedure allows physically meaningful and reproducible results. We have good and exhaustive experiences with Jang and Allebach's method; it is robust and measures reasonable MTFs. Thus we use the measurements resulting from this method in the next sections.

\section{Compensating for the Printer Modulation Transfer Function}

The initial aim of this study was to be able to compensate for the printer MTF by preprocessing the image sent to the printer, as depicted in Fig. 25. The compensation of an MTF is already known from other fields as the restoration of blurred images. ${ }^{17}$ For example, consider a picture of a real scene captured with a digital camera: as cameras have a low-pass characteristic, the captured image is not sharp. The MTF of the camera can be compensated to obtain a sharper image, closer to the real scene. While camera compensation occurs in postprocessing, printer compensation must occur in preprocessing. Nevertheless, it is based on the same principles, since both processes aim to amplify the frequencies by a factor given by the system MTF.

Another critical difference is that printing systems are nonlinear. Therefore, an optimal compensation of the MTF should depend on different parameters related to the characteristics of the input images such as the bias, color, spatial frequency, or orientation.

\subsection{Deconvolution}

Under the assumption of a linear system, the degradation can be described by a multiplication in the Fourier domain. Assuming an input image $\mathbf{I}_{\text {in }}$, its Fourier transform being $F\left(\mathbf{I}_{\text {in }}\right)$ and the system modulation transfer function MTF, the Fourier transform of the degraded image at the output is $F\left(\mathbf{I}_{\text {out }}\right)=\mathrm{MTF} \times F\left(\mathbf{I}_{\text {in }}\right)$. In the case where MTF is known at each frequency, the compensation is a simple division in the Fourier domain and the compensated input image $\mathbf{I}_{\text {comp }}$ is then obtained by the inverse Fourier transform $F^{-1}$ as follows:

$$
\mathbf{I}_{\text {comp }}=F^{-1}\left[\frac{F\left(\mathbf{I}_{\text {in }}\right)}{\mathrm{MTF}}\right] .
$$

This is also known as deblurring or as a deconvolution, since it is the inverse of a convolution in the spatial domain. Unfortunately this ideal case might be perturbed by noise. The system MTF has to be estimated by a measurement which itself is affected by noise. This is mostly problematic for frequencies where the system attenuation is strong, leading to low values of the MTF and to numerical instabilities in the deconvolution. Furthermore, the image $\mathbf{I}_{\text {in }}$ might also contain noise. Different filters have been proposed to take the noise into account. ${ }^{18}$

\subsection{Wiener Filter}

The Wiener filter named after its inventor ${ }^{19}$ is a modification of the division in Eq. (11), taking the noise into account. It is done by dividing through the sum of the MTF and a noise-to-signal ratio:

$\mathbf{I}_{\text {comp }}=F^{-1}\left(\frac{F\left(\mathbf{I}_{\text {in }}\right)}{\mathrm{MTF}} \times \frac{\mathrm{MTF} \times \mathrm{MTF}^{*}}{\mathrm{MTF} \times \mathrm{MTF}^{*}+\mathrm{NSR}}\right)$,

where $\mathbf{I}_{\text {in }}$ is the input image, $\mathbf{I}_{\text {comp }}$ is the compensated image, MTF is the MTF of the system, MTF* is its complex conjugate, and NSR is the noise-to-signal ratio of the image $\mathbf{I}_{\text {in }}$. When the noise tends to zero, the Wiener filter reduces to Eq. (11): But the more noise there is in the image, the less the filter impact. The estimation of the noise-to-signal ratio NSR might be problematic, as a printer can get an input image from any source (camera, scanner, software, etc.) and every device has its own noise characteristics.

There is a variety of other possibilities to compensate a convolution kernel using different techniques (see Chan and Shen ${ }^{18}$ for an overview). One issue with all these advanced methods is that they tend to use more calculation time and storage than the simple division from Eq. (11).

\subsection{Unsharp Masking}

Alternatively, unsharp masking, ${ }^{20}$ based on the subtraction of a smoothed image from the input image, can be used to increase contrast of existing edges:

$\mathbf{I}_{\text {comp }}=\mathbf{I}_{\text {in }}+\alpha \times\left[\mathbf{I}_{\text {in }}-f_{\text {blur }}\left(\mathbf{I}_{\text {in }}\right)\right]$,

where $f_{\text {blur }}$ is a blurring low-pass filter such as a Gaussian filter, and $\alpha$ is a constant coefficient setting the amount of unsharp masking. This linear filter is frequently used in the digital photography community, usually to regain sharpness 


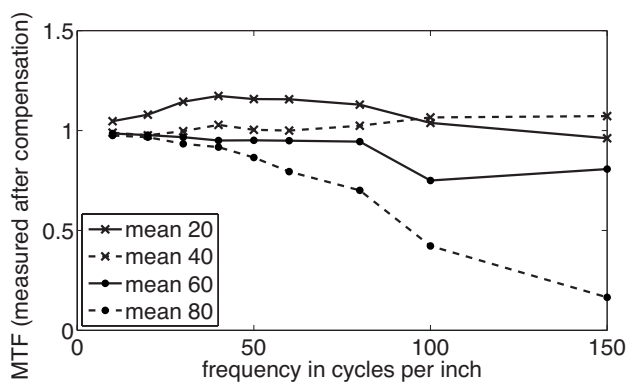

(a)

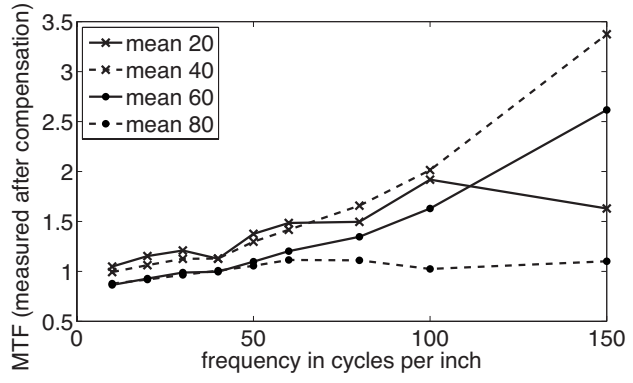

(b)

Fig. 26 MTF measured after compensation by a simple deconvolution. Four rows from the Jang and Allebach test page have been compensated using two different sets of MTF values: in (a) with MTF values from an average of midtone gray rows, and in (b) an average of highlight gray rows.

attenuated in the scanning process, or sharpen reproduction of images on prints. Limitations of such filters include the need to set a filter size in $f_{\text {blur }}$ that limits the gain of sharpness to a fixed range of frequencies. An unapropriate setting of the size might lead to halos in resulting images. Furthermore, $\alpha$ is a constant coefficient setting the amount of boosting uniformly across the fixed frequency range, whereas frequency-dependent coefficients would allow a more effective compensation of the MTF.

\subsection{Experiment: Applying a Simple Deconvolution}

The goal of the MTF compensation is to obtain a compensated $\mathrm{MTF}=1$ across frequencies and bias levels. Deconvolution does allow a compensation across frequencies, yet the measured MTF depends not only on the frequency but also on the bias level. Thus deciding how to set the deconvolution for the whole range of bias levels is not easy. In preliminary tests, we attempted to use the mean of MTFs over the gray bias. ${ }^{21}$ Consequently, the measured compensated MTFs were too low (undercompensated) for light gray patches and too high (overcompensated) for dark gray patches. To demonstrate the problem, we generate four rows from Jang and Allebach's test page with different mean values $\left(Y_{\text {mean }} \in\{20,40,60,80\}\right)$. These rows are then compensated using two different sets of MTF values. In one workflow, we use MTF values measured from sine waves in the midtone grays (average of rows 9 to 11 from Fig. 18). In the following we name it a weak compensation. In another workflow, we use MTF values measured from sine waves from the highlight grays (average of rows 17 to 19). In the following we name it a strong compensation. The compensated test pages are then printed and scanned, as described in Sec. 5. The compensated MTF values are measured and reproduced in Fig. 26. This figure shows the measured MTF values resulting from the compensation with MTF values from the midtone grays [Fig. 26(a)] and the highlight grays [Fig. 26(b)]. The results of the compensation with the MTF values from the midtone grays in Fig. 26(a) show clearly that the compensation is not strong enough for the highlight grays (the mean 80 values), as the resulting MTF values are still well under the optimal value $\mathrm{MTF}=1$. The higher the bias (or mean) level, the lower the MTF values of the compensated test image. The reason is that the MTF, which has been used for the compensation, is resulting from measures from the midtones and thus is higher than the MTF measures in the highlights. Therefore the compensation does not sufficiently boost the sinusoidal waves in the highlights. Accordingly in Fig. 26(b), sinusoidal waves in the shadows (the mean 20 values) and midtones (mean 20 and 60) are overcompensated when using MTF values from the highlights for the compensation.

These results clearly show that the compensation works if and only if an MTF with an appropriate bias value is used for the deconvolution. But since image brightness values usually cover a large range of values, this simple deconvolution will not give satisfying results. Thus, a more sophisticated algorithm has to be used to account for different bias levels within a given image.

\section{Locally Adaptive Compensation}

In this study, preliminary tests showed that the compensation based on a simple division gives satisfying results in restricted cases. Such good results are mainly due to the fact that the measured MTF values are mostly greater than 0.5 . Therefore, we propose to use simple division and extend it by taking into account the nonlinearity of the system.

The MTF values vary as a function of the mean gray level: Fig. 18 shows how the MTF values in the highlights significantly differ from the ones in the midtones and the shadows.

In this section we propose a compensation algorithm based on image decomposition, which locally adjusts the MTF compensation in an image given the local mean for each pixel of that image. Since the HVS is more sensitive to luminance high-frequency content than chrominance high-frequency content, we limit the deconvolution to grayscale high-frequency content of the image. Furthermore, to avoid the introduction of halos, ${ }^{22}$ we first decompose the image in two bands in the CIELAB color space, the highpass band $\mathbf{I}_{\text {high }}$ and the low-pass band $\mathbf{I}_{\text {low }}$. The compensation algorithm is then applied to $\mathbf{I}_{\text {high }}$ only.

\subsection{Image Decomposition}

One key aspect of the proposed mathematical framework is the decomposition of the image in two bands. The goal of this decomposition is to set apart the local means and the local details of the image to process them separately and preserve both as much as possible in the resulting image. Such image decomposition relies on filters such as Gauss- 
ian filters. One issue with Gaussian-based decomposition is that halos are often found in the resulting images: Kimmel et al., ${ }^{23}$ Morovič and Wang, ${ }^{24}$ and Balasubramanian, Queiroz, and Eschbach ${ }^{25}$ all acknowledge halos. It is critical to address these halos that degrade the image: Morovič and Wang adjusted the filter sizes and the number of decomposition bands to contain them. Kimmel et al. used a feature mask similar to the one we proposed in Bonnier and Simoncelli. ${ }^{26}$ Farup, Gatta, and Rizzi ${ }^{27}$ proposed adaptive operators to limit the halos. In accordance with Zolliker and Simon $^{28}$ and Kolas and Farup ${ }^{29}$ we believe that an edge preserving filter will help avoid halos. The 5-D bilateral filter $(\mathrm{BF})$ is probably the most adapted edge preserving filter. The size of the filter is a crucial parameter and was thoroughly investigated by Bonnier. ${ }^{10}$ The number of frequency bands in the decomposition is also considered with a minimum of two bands and a maximum only constrained by the cost of the computation time.

The decomposition is obtained by 5-D BF in the CIELAB space as proposed by Tomasi and Manduchi. ${ }^{30}$ It is a combined spatial domain and color range filtering. Let $L_{\text {low }}^{*}=\mathrm{BF}\left(L^{*}\right)$ denote the $L^{*}$ channel of the filtered image. The $L_{\text {low }}^{*}$ value of pixel $i, L_{\text {low }}^{* i}$, can be obtained as follows:

$L_{\mathrm{low}}^{* i}=\sum_{j \in \mathbf{I}_{\mathrm{in}}} w_{\mathrm{BF}}^{j} L^{* j}, \quad w_{\mathrm{BF}}^{j}=\frac{d\left(\mathbf{x}^{i}, \mathbf{x}^{j}\right) r\left(\mathbf{p}^{i}, \mathbf{p}^{j}\right)}{\sum_{j \in \mathbf{I}_{\mathrm{in}}} d\left(\mathbf{x}^{i}, \mathbf{x}^{j}\right) r\left(\mathbf{p}^{i}, \mathbf{p}^{j}\right)}$,

where $\mathbf{I}_{\text {in }}$ is the input image, $d\left(\mathbf{x}^{i}, \mathbf{x}^{j}\right)$ measures the geometric closeness between the locations $\mathbf{x}^{i}$ of pixel $i$ and $\mathbf{x}^{j}$ of a nearby pixel $j, r\left(\mathbf{p}^{i}, \mathbf{p}^{j}\right)$ measures the colorimetric similarity between the colors $\left(L^{* i}, a^{* i}, b^{* i}\right)$ and $\left(L^{* j}, a^{* j}, b^{* j}\right)$ of pixels $i$ and $j$.

In our implementation, $d\left(\mathbf{x}^{i}, \mathbf{x}^{j}\right)$ and $r\left(\mathbf{p}^{i}, \mathbf{p}^{j}\right)$ are Gaussian functions of the Euclidean distance between their arguments:

$$
\begin{gathered}
d\left(\mathbf{x}^{i}, \mathbf{x}^{j}\right)=\exp \left[-\frac{1}{2}\left(\frac{\left\|\mathbf{x}^{i}-\mathbf{x}^{j}\right\|}{\sigma_{d}}\right)^{2}\right], \\
r\left(\mathbf{p}^{i}, \mathbf{p}^{j}\right)=\exp \left[-\frac{1}{2}\left(\frac{\Delta E_{a b}^{*}\left(\mathbf{p}^{i}, \mathbf{p}^{j}\right)}{\sigma_{r}}\right)^{2}\right],
\end{gathered}
$$

where the two scale parameters $\sigma_{d}$ and $\sigma_{r}$ play an essential role in the behavior of the filter. In classic Gaussian filtering, the width of the Gaussian (set by $\sigma_{d}$ ) determines the boundary between the lower frequency content going to the low-pass band (considered as local means) and the higher frequency content going to the high-pass band (local details). Setting the appropriate value for $\sigma_{d}$ is not a trivial task. This choice relates to the definition of "local details" (i.e., small or minor elements in a particular area). This definition depends on multiple parameters such as the size and resolution of the reproduction, the modulation transfer function of the reproduction device, the viewing conditions, the distance of visualization, and the behavior of the human visual system. The human visual system is often modeled by multiscale decompositions ${ }^{31}$ with more than two bands (usually up to five).

Such multiscale decomposition has been proposed in several papers. ${ }^{10}$ It could be relevant in our algorithm and would allow the definition of several categories of details with different sizes. However, for the sake of keeping the algorithm simple and the computing cost low, we limit the image decomposition to two bands. Thus we need to investigate the impact of $\sigma_{d}$ on the decomposition to select an appropriate value.

In the 5-D bilateral filter, the $\Delta E_{a b}^{*}$ color distance between the central pixel and nearby pixels is also taken into account. This allows us to avoid halos and handle specifically the local transitions between local similar pixels. Nearby pixels at small $\Delta E_{a b}^{*}$ distance (i.e., perceived as similar) are filtered. Pixels are less and less filtered as the $\Delta E_{a b}^{*}$ distance becomes large compared to $\sigma_{r}$. Thus $\sigma_{r}$ determines a reference to set apart small $\Delta E_{a b}^{*}$ from large $\Delta E_{a b}^{*}$. While small $\Delta E_{a b}^{*}$ values are well correlated with perceived color differences, it is more difficult to define a threshold $\sigma_{r}$ above which $\Delta E_{a b}^{*}$ values can be considered as large. Given the lack of a straightforward definition for "local details" and "similar colors," we propose to first review the previous work and then evaluate the impact of $\sigma_{d}$ and $\sigma_{r}$ values on the image decomposition.

Tomasi and Manduchi ${ }^{30}$ explore different values for $\sigma_{d}$ and $\sigma_{r}$, and present 8-bit grayscale images processed with $\sigma_{d}=3$ pixels and $\sigma_{r}=50$, yet the sizes of the processed images are not specified. As the setting of $\sigma_{d}$ should depend on the image size and the conditions of visualization, Zolliker and Simon ${ }^{32}$ obtained good results with $\sigma_{d}$ in the range of [2 to 5$] \%$ of the image diagonal and $\sigma_{r}$ values in the range of [10 to 25] $\Delta E_{a b}^{*}$. They have applied the filter in their spatial gamut mapping algorithm with $\sigma_{d}=4 \%$ of the image diagonal and $\sigma_{r}=20 \Delta E_{a b}^{*}$.

In the first implementation of our algorithm, we empirically set the values to $\sigma_{d}=1 \%$ of the image diagonal and $\sigma_{r}=25 \Delta E_{a b}^{*}$ (for images printed at $150 \mathrm{dpi}$, at the size [9 to 15$] \mathrm{cm}$ by [12 to 20$] \mathrm{cm}$, viewed at a distance of $60 \mathrm{~cm}$ ). This value $\sigma_{d}=1 \%$ of the image diagonal is not in the range proposed by Zolliker and Simon, but the context and the filtered images are different: they filter image differences and we filter the whole image. This means that the characteristics (contrast, saturation, etc.) are different and the settings of the bilateral filter may consequently differ. A larger value of $\sigma_{d}$ means a broader filter in the image domain, thus a larger set of frequencies being filtered. A larger value of $\sigma_{r}$ means a larger filter in the color domain, thus a larger range of color transitions being filtered. When $\sigma_{r}$ is very large, the bilateral filter is not modulated by the color content of the filtered area, and the resulting blurring of the image becomes similar to the blurring of a two-dimensional Gaussian filter. It also leads to the introduction of halos near the strong edges. A small value of $\sigma_{r}$ severely limits the blurring of the image to very small color transitions for any $\sigma_{d}$. A small value of $\sigma_{d}$ limits the blurring of the image to high-frequency content for any $\sigma_{r}$. When both $\sigma_{d}$ and $\sigma_{r}$ have very large values, $\mathbf{I}_{\text {low }}$ shows some color shifts due to a large boost of chroma in desaturated areas surrounded by saturated areas.

Based on the observations in Bonnier et al. ${ }^{33}$, in our experiments the parameters are set to $\sigma_{d}=4 \%$ of the image diagonal and $\sigma_{r}=20 \Delta E_{a b}^{*}$. To compute the low-pass band $\mathbf{I}_{\text {low }}$, only the channel $L_{\text {in }}^{*}$ of the input image $\mathbf{I}_{\text {in }}$ is filtered using 5-D bilateral filtering as described before [Eqs. (14) 


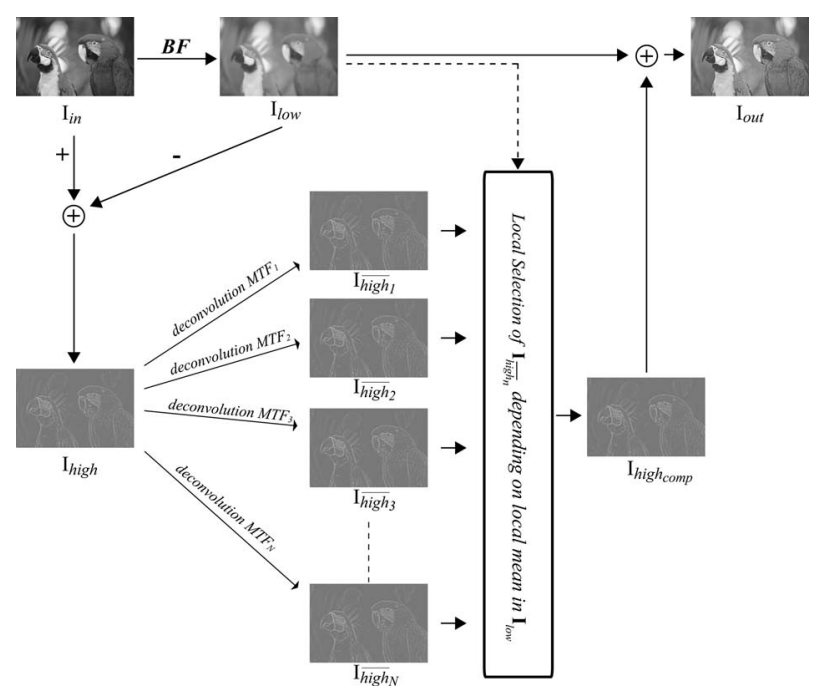

Fig. 27 Principle of the locally adaptive MTF compensation: the high-pass band $I_{\text {high }}$ is compensated $N$ times for $\mathrm{MTF}_{n}$. Then for each pixel, a locally adaptive merging is processed according to the local mean estimated from $\mathbf{I}_{\text {low. }}$.

and (15)]. Nevertheless, since the 5-D bilateral filter involves the $\Delta E_{a b}^{*}$ distance, the $a^{*}$ and $b^{*}$ values are taken into account in the filtering of $L_{\text {in }}^{*}$. The high-pass band $\mathbf{I}_{\text {high }}$ is then calculated by taking the difference of $\mathbf{I}_{\text {in }}$ and the low-pass band $\mathbf{I}_{\text {low }}$ :

$\mathbf{I}_{\mathrm{low}}=\left(L_{\mathrm{low}}^{*}, a_{\mathrm{in}}^{*}, b_{\mathrm{in}}^{*}\right)$,

$\mathbf{I}_{\text {high }}=\mathbf{I}_{\text {in }}-\mathbf{I}_{\text {low }}=\left(L_{\text {in }}^{*}-L_{\text {low }}, 0,0\right)$,

with $L_{\text {low }}^{*}=B F\left(L_{\text {in }}^{*}\right), L_{\text {high }}^{*}=L_{\text {in }}^{*}-L_{\text {low }}^{*}$.

\subsection{Bias Dependent Compensation}

We propose to take into account the image local mean gray level as an estimation of the bias when compensating for the printer MTF: each pixel in $L_{\text {high }}^{*}$ is compensated according to the local mean in $\mathbf{I}_{\text {in }}$ :

$L_{\text {high }_{\text {comp }}^{*}}^{*}=\operatorname{comp}_{\mathrm{MTF}}\left(L_{\text {high }}^{*}, \mathbf{I}_{\mathrm{in}}, \mathrm{MTF}\right)$,

where $L_{\text {high }_{\text {comp }}}^{*}$ is the compensated $L^{*}$ channel and comp MTF $_{\text {. }}$ the locally adaptive compensation (see Fig. 27).

\subsection{Local Mean}

A local measure of the mean gray level $L_{\text {in }}^{*}$ is needed to achieve the locally adaptive compensation. Here we can take full advantage of the image decomposition and use $\mathbf{I}_{\text {low }}$ as an estimation of the local mean. Notice that values in $\mathbf{I}_{\text {low }}$ are obtained by bilateral filtering. This is an advantage, as a local mean computed in $\mathbf{I}_{\text {in }}$ for a local detail in $\mathbf{I}_{\text {high }}$ should only be computed by using local and similar gray pixels, as this is done in $\mathbf{I}_{\text {low }}$ by the bilateral filter. Since the MTF was measured using modulation and bias values based on the CIE XYZ color space, $\mathbf{I}_{\text {low }}$ is converted to CIE $\mathrm{XYZ}$ and $Y_{\text {low }}$ is taken as the local mean:
$L_{\text {high }_{\text {comp }}^{*}}^{*}=\operatorname{comp}_{\mathrm{MTF}}\left(L_{\text {high }}^{*}, Y_{\text {low }}, \mathrm{MTF}\right)$.

\subsection{N Deconvolutions of $L_{\text {high }}^{*}$}

The MTF has been measured for the bias values $b_{n}, n$ $\in[1, N]$. Let $\mathrm{MTF}_{n}$ denote the MTF measured for bias $b_{n}$. $L_{\text {high }}^{*}$ can be divided through each $\operatorname{MTF}_{n}(f)$ in the Fourier domain, which results in $N$ compensated high-pass images:

$L_{\mathrm{high}_{n}}^{*}=F^{-1}\left[\frac{F\left(L_{\text {high }}^{*}\right)}{\mathrm{MTF}_{n}}\right], \quad n \in[1, N]$,

where $L_{\mathrm{high}_{n}}^{*}$ is the compensated image in the image domain for bias value $b_{n}$.

In this study, we have not considered the orientation of the high-frequency content, as the measured MTF of our printing system (Océ Colorwave 600) is close to being orientation independent. In our implementation, the compensation is applied in the Fourier domain as a division by a 2-D filter constructed by a 360-deg polar rotation of the 1-D MTF

Yet for most of the printing systems, the measured MTF of a printing system depends on the orientation. In such a case, the MTF shall be measured at different orientations. The compensation is then easily expandable by constructing the 2-D filter using the measured MTF at the corresponding angles and interpolation for intermediate angles.

\subsection{Adaptive Merging}

Locally adaptive merging can now be processed for each pixel according to the local mean. The $N$ compensated high-pass bands $L_{\overline{\text { high }}_{1}}^{*}, \ldots, L_{\mathrm{high}_{N}}^{*}$ are merged to one single compensated high-pass band $L_{\text {high }}^{*}$. Sinp Since only some of the MTF values have been measured for a limited set of bias and frequency values, missing MTF values are first linearly interpolated for the frequency range of the image sent to the printer $([0,150]$ dpi in our experiments). Then for each pixel in $\mathbf{I}_{\text {high }}$, the two bias levels $b_{n}$ and $b_{n+1}$ closest to the value $Y_{\text {low }}$ corresponding to that pixel are selected: $b_{n} \geqslant Y_{\text {low }} \geqslant b_{n+1}$. $L_{\text {high }}^{*}$ is a linear interpolation between the compensated images $L_{\text {high }_{n}}^{*}$ at these two bias levels:

$L_{\text {high }_{\text {comp }}^{*}}^{*}=w \cdot L_{\text {high }_{n}}^{*}+(1-w) \cdot L_{\text {high }_{n+1}}^{*}$,

with

$w=\frac{Y_{\text {low }}-b_{n+1}}{b_{n}-b_{n+1}}$.

\subsection{Experimental Results}

In this section we assess the locally adaptive compensation. As in Sec. 7.4, the four rows from Jang and Allebach's test page with different means $\left(Y_{\text {mean }} \in\{20,40,60,80\}\right)$ are compensated, this time with the new locally adaptive method. The resulting MTF measurements of the four compensated images are shown in Fig. 28(b). To better visualize the improvement, the results from the strong compensation in Fig. 26(b) have been reproduced in Fig. 28(a) 


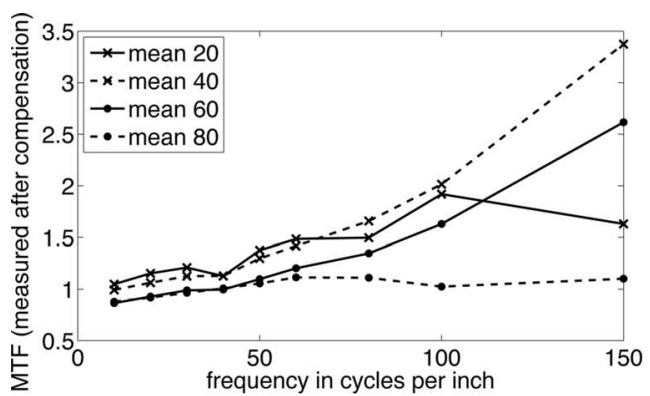

(a)

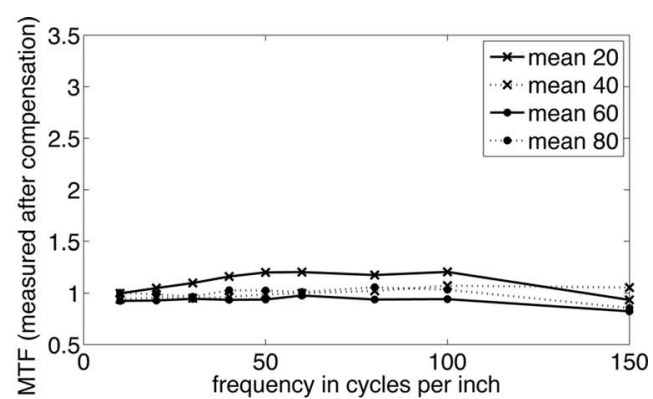

(b)

Fig. 28 (b) MTF measurements obtained with locally adaptive compensation as explained earlier. (a) has been reproduced from Fig. 26(b) for comparison.

(both MTF axes have the same range). In comparison to the results from the simple compensation, the measured values are very close to the optimal case where all the MTF values are equal to 1 over the whole frequency and bias ranges.

These results clearly show that an image with different local gray levels can be successfully processed so that the resulting transfer function of the entire printing system (measured with our modified version of Jang and Allebach's method) is close to $\mathrm{MTF}=1$. These results also show that the proposed locally adaptive compensation is consistent with the used measurement method.

\section{Discussion}

In this section we first compare the proposed algorithm to existing alternatives, then discuss possible evolutions.

\subsection{Locally Adaptive Compensation Compared to Classic Deconvolution and Unsharp Masking}

First, unlike the classic deconvolution and unsharp masking, the proposed locally adaptive compensation is based on image decomposition and thus is only applied to a carefully restricted set of high-frequency content. This allows the results of the proposed algorithm to be free of haloing artifacts. Secondly, the proposed compensation is bias dependent, the MTF compensation is pixel-wise optimized to produce a resulting image with a constant $\mathrm{MTF}=1$ across the printed image. To obtain similar results with unsharp masking, multiple filters with decreasing size of blurring filters and different amounts of unsharp masking would be needed. While theoretically possible, this solution is obviously neither as elegant nor as precise as the proposed algorithm.

\subsection{Optimization}

It should be possible to reduce the number of bias levels used within the compensation by using nonlinear weights in Eq. (21). These weights would be deduced from the bias dependency of the printer MTF. The approach of reducing the number of bias levels and therefore using a nonlinear interpolation would be interesting, since it would reduce the number of inverse Fourier transforms within our compensation algorithm.

It could even be profitable to use MTFs from bias levels that are not equally spaced. For example, fewer bias levels in gray level ranges where the bias dependency is weak and more in ranges where the bias dependency is strong. How to efficiently choose the number and values of the bias levels used for the compensation and how to interpolate between them is an optimization problem and would aim at providing full quality with as little computation power as possible.

\section{Overcompensation}

In the previous section, the compensation of the MTF was based on objective measures by means of a scanner. According to the scanner-based measured objective results in Sec. 8.6, the MTF compensation has achieved its goal: the MTF of the compensated system is measured as being much closer to 1 by the scanner.

Yet preliminary evaluation of the printed images by observers lead to interrogations about the amplitude of the perceived improvement. The broader goal of this research is to improve the quality of printed images by preserving the perceived image content. To achieve this goal, the amplitudes of the image features should be perceived as identical to the amplitudes perceived when the same image is displayed on a monitor. It is well known that the perception of the image features depends on the human visual system (HVS), the medium characteristics (color temperature and luminance level of the white point, luminance level of the black point, color gamut), and the viewing conditions. ${ }^{34}$ Therefore, the comparison of an image reproduced on a monitor with the same image printed with different characteristics and different viewing conditions will be impacted by these differences. For example, a lesser global contrast might alter the perception of local contrast.

To counter this impact and further enhance the perceived quality of the reproduction, it might be necessary to overcompensate the MTF. In this scenario (see Fig. 29), the MTF of the printing system is compensated for more than necessary, and a stronger local contrast should be perceived in the resulting images. Such images could show better preserved local details and thus be judged as more accurate reproductions.

\subsection{How to Overcompensate}

Unfortunately, it is difficult to know how overcompensation should be applied, as it depends on the perception of the HVS. In our tests, we have thought of overcompensating as 


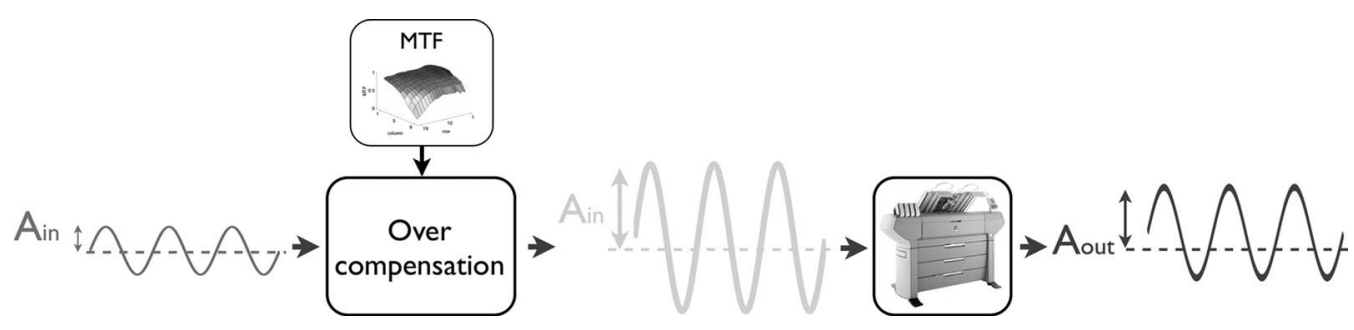

Fig. 29 Principle of the MTF overcompensation: the amplitude $A_{\text {in }}$ is overcompensated (overpreamplified) by overcompensation (division by the MTF in the Fourier domain) before printing, resulting in an output amplitude $A_{\text {out }}$ larger than the input $A_{\text {in. }}$.

an underestimation of the printer MTF. Several operators can be applied to lower the MTF values, resulting in an overcompensation of the MTF:

- A global shift: $\mathrm{MTF}_{\text {over }}=\max (\mathrm{MTF}-\beta, 0)$, where $\mathrm{MTF}_{\text {over }}$ is the modified MTF value and $\left.\left.\beta \in\right] 0,1\right]$ is the shifting coefficient. This shifting will have a stronger impact on high-frequency content.

- A global scaling factor: $\mathrm{MTF}_{\text {over }}=\theta \times \mathrm{MTF}$, where $\mathrm{MTF}_{\text {over }}$ is the modified MTF value and $\left.\left.\theta \in\right] 0,1\right]$ is the scaling coefficient. Scaling will have a stronger impact on low-frequency content.

- A frequency dependent function, such as power applied to the MTF values, might also have an interresting behavior.

\subsection{Proposed Overcompensation}

Psychophysical experiments should be carried out to gain knowledge about the perception of overcompensation. Preliminary tests show that a global scaling factor has a stronger perceived impact. An underestimation of $20 \%$ of the MTF, obtained by multiplying each MTF value by $\theta=0.8$, has been selected for its perceptually satisfying results.

\section{Psychophysical Experiment}

The goal of this experiment was to evaluate the gain of the MTF compensation in a printing workflow using spatial and color adaptive gamut mapping algorithms. ${ }^{10,35}$ To evaluate the compensation and overcompensation of the printer MTF, three alternative degrees of compensation have been included in this study:

1. no compensation

2. locally adaptive MTF compensation

3. locally adaptive MTF overcompensation obtained by reducing the measured MTF by $20 \%$ by multiplying it by $\theta=0.8$, leading to compensation by a factor $1 / \theta=1 / 0.8=1.25$, or $25 \%$.

\subsection{Experiment Type and Observer Task}

In this category judgment experiment, the observers were presented three samples of a series of processed images along with a reference reproduction. They have to evaluate how close each reproduction is to the reference reproduction, on a scale of numbers from 1 to 7 , where 1 represents the closest reproduction you can imagine and 7 represents the least accurate reproduction possible. A description of the accuracy for each level was proposed to help: 1. most accurate you can imagine, 2 . highly accurate, 3 . very accurate, 4. fairly accurate, 5. moderately accurate, 6. poorly accurate, and 7. least accurate reproduction possible. It was suggested to make the decision based on different parts of the image, to evaluate the fidelity of the reproduction of both colors and details, and look for possible artifacts. It is the accuracy of reproduction of the images that is evaluated, not the pleasantness.

\subsection{Images}

A total of 15 images was used in this experiment: seven sRGB images from the ISO 12640-2:2004 standard, ${ }^{36}$ and eight CIELAB/SCID images from the ISO $12640-3 .^{37}$ (reproduced in Fig. 30). Images were printed at a resolution of 150 pixels per inch and a size of $1200 \times 1500$ pixels, $20.32 \times 25.4 \mathrm{~cm}\left(\right.$ i.e., $8 \times 10$ in.). ${ }^{10}$

\subsection{Color Processing}

The destination gamut was the gamut of an Océ ColorWave 600 printer using Océ Red Label uncoated paper and the printer at the highest quality setting Présentation mode. It was measured by a GretagMacbeth spectrophotometer using GretagMacBeth MeasureTool 5.0.8 (GretagMacBeth, Grand Rapids, Michigan). The sRGB images were converted to CIELAB using the relative colorimetric intent of the sRGB profile. The CIELAB/SCID images are natively encoded in the CIELAB color space. All the images were first gamut mapped to the gamut of the output printing system using the spatial and color adaptive compression (SCACOMP) algorithm. Then they were compensated as described in Sec. 8. The resulting images were then converted from CIELAB to the device CMYK using the relative colorimetric intent of a custom printer CMYK ICC version 2 profile. They were printed on a Océ ColorWave 600 printer using Océ Red Label paper with the color management disabled.

\subsection{Observers and Viewing Conditions}

The test panel was constituted by eight female and nine male observers with normal vision and various degrees of expertise in judging image quality. The five printed gamutmapped candidates were presented in a controlled viewing room with a lighting system at a color temperature of $5200 \mathrm{~K}$, an illuminance level of $450 \mathrm{lux} \pm 75$, and color rendering index of 96 . Our aim was to carry a thorough evaluation by having two sets of images with different input color gamuts and reference reproduction devices. The 

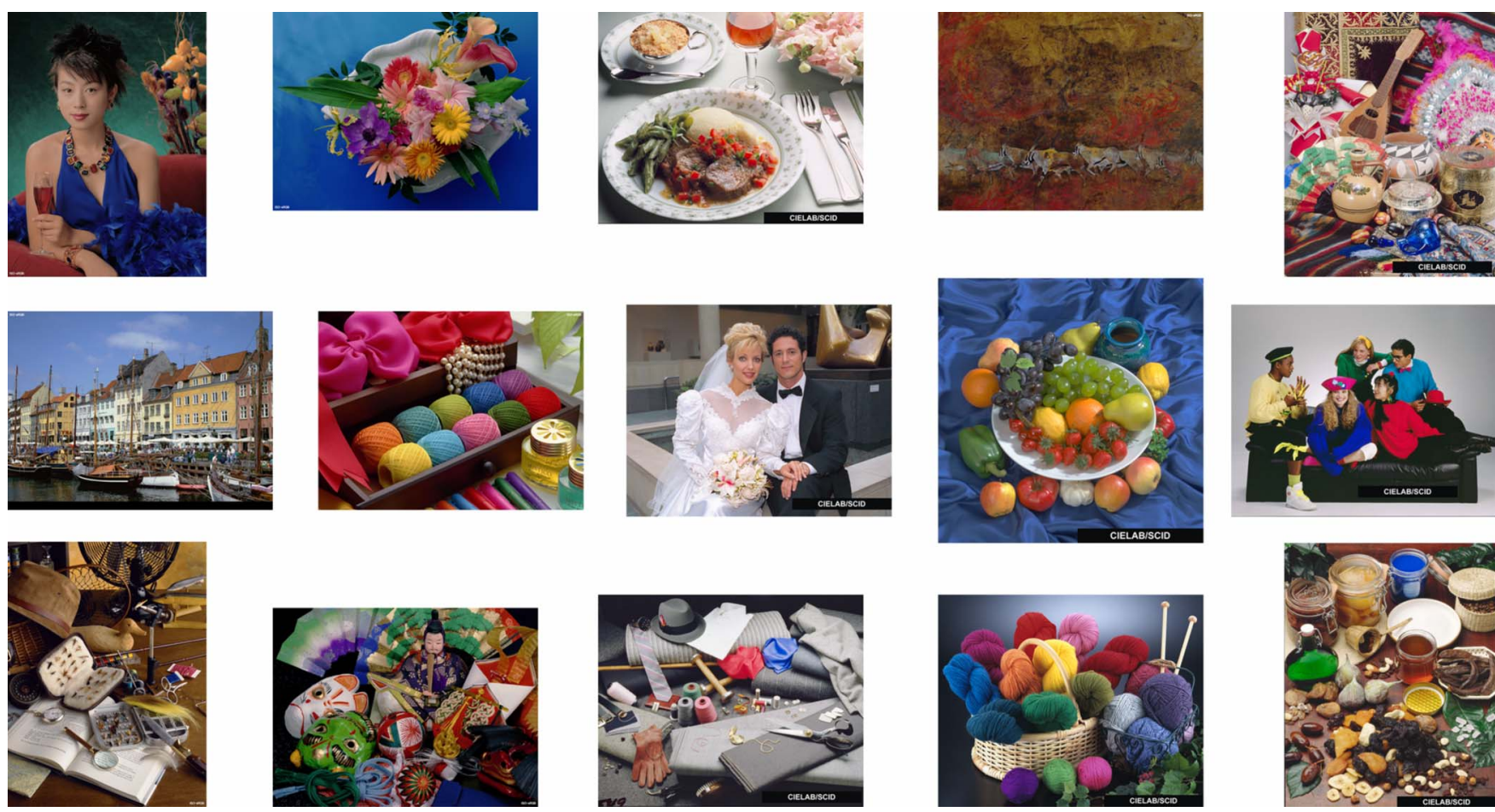

Fig. 30 sRGB images form ISO 12640-2 and CIELAB/SCID images from ISO 12640-3.

observers viewed simultaneously the reference image and the printed images from a distance of approximately $60 \mathrm{~cm}$. The experiment was then two-fold.

- For the sRGB image set, the observers were presented with a reference image on an EIZO ColorEdge CG221 display (EIZO, Ishikawa, Japan) at a color temperature of $5200 \mathrm{~K}$ and luminance level of $120 \mathrm{~cd} / \mathrm{m}^{2}$. This set is rendered for sRGB display. Therefore a monitor capable of displaying the sRGB gamut is the most compatible reproduction device for this set of images.

- For the CIELAB/SCID image set, the observers were presented with a reference image printed on an Epson R800 printer using Epson Premium Glossy Photo Paper in the best printing quality mode. This set is rendered for the perceptual reference medium gamut (PRMG). The most appropriate way to reproduce these images is using a printing system able to reproduce the color gamut PRMG and with good capability to reproduce details, such as the Epson R800 printer with Epson Premium Glossy Photo Paper.

\subsection{Global Results}

Results cumulated over the 15 images and 17 observers in Fig. 31 show that overall the overcompensation outperforms other alternatives. Each version is perceived as significantly different from the others, from the top rated overcompensation, then compensation, and no compensation. Mean scores for overcompensation and compensation are in category 3, mean score for no compensation is in category 4. Overall, images with MTF compensation were evaluated as more accurate than without, and the overcompensated version was given the best scores. Several observ- ers also reported that, overall, the printed overcompensated images appear to have a better preserved local contrast when compared to noncompensated and compensated reproductions of the same images. This is a clear indication that MTF compensation fulfills its goal of producing more accurate reproductions. Further study will be necessary to find an optimal overcompensation operator in such workflows.

In this experiment we assessed the opportunity of using MTF compensation in our printing workflow. In such a workflow, the viewing conditions and gamuts are different for the images displayed on the monitor and the ones printed. This is usually the case in printing workflow, and such differences might lead the observers to select the overcompensated images more than the compensated ones. The results might also be due to an overestimation of the MTF by Jang and Allebach's method, or by an underevaluation of the MTF by the human visual system. In both cases, it would probably be due to the local averaging achieved to

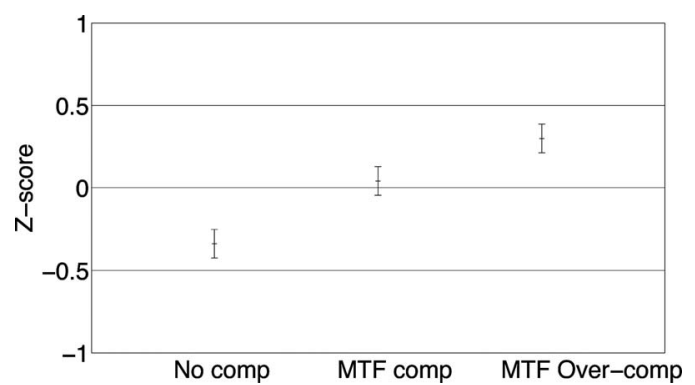

Fig. 31 Z-scores resulting from our experiment (category scaling), averaged over 15 images and 17 observers. 
convert halftoned images to continuous tone images (see also Sec. 2.2). If we were able to match the viewing conditions and gamuts of the displayed and printed images, we would have the opportunity to evaluate the compensation of the MTF by verifying that the compensated images would be evaluated by observers as the closest reproduction to the reference reproduction.

\section{Conclusions}

We provide a comparison of three printer MTF measurement methods. The comparison shows that the slanted edge method and Jang and Allebach's method measure comparable MTF values, while MTF values from Hasegawa et al.'s method are significantly lower.

The method from Jang and Allebach is more complete than the others, since it additionally measures the bias dependency of the MTF values. This dependency is significant for our inkjet printing system Océ Colorwave 600, and the additional measurements can be exploited for printeradapted image processing based on the MTF measurement. We present new improvements to the MTF measurement method proposed by Jang and Allebach. We propose to use the $Y$ value of CIE XYZ in the test target instead of device RGB and in the analysis instead of a $\Delta E_{a b}^{*}$ measure in CIELAB. This improvement not only avoids the systematic error discussed in Ref. 14, but also simplifies the implementation of the method. Future research on MTF measurement methods should investigate the reliability of the method with different devices (printing systems and scanners) and measurements with color inks.

In the second part of this work, we propose an approach to compensate for the printer modulation transfer function. A new compensation algorithm is presented. It fully exploits both the frequency and bias dependency of the measured MTF values. The category judgment experiment on photographic images delivers encouraging results: MTF compensation fulfills its goal of producing more accurate reproductions. More studies remain necessary to fully understand the benefits and drawbacks of MTF compensation and to find optimal parameters for overcompensation, including the case of noisy images. In a printing workflow composed of an input device (such as a scanner/digital camera or a monitor) and an output system (such as a printing system or a monitor), MTF compensation should consider the MTFs of both devices and process the captured image once to compensate for the two MTFs. The use of MTF compensation in other imaging workflows should also be investigated.

\section{Acknowledgments}

First and foremost, the authors thank their supervisor, the late Francis Schmitt (Telecom ParisTech, France), who was abundantly helpful and offered invaluable assistance, support, and guidance. The authors also want to thank Océ Print Logic Technologies for supporting this research. Many thanks especially to Marie Dubail (ENS Louis Lumiere, Paris, France), Frans Gaykema (Océ R \& D, Venlo, The Netherlands) for his help and good advice, and to Isabelle Bloch (Telecom ParisTEch, France) for her thoughtful comments.

\section{References}

1. J. J. McCann, "Color gamut mapping using spatial comparisons," Proc. IS\&T/SPIE 4300, 126-130 (2009).

2. P.-L. Sun, "The influence of image characteristics on colour gamut mapping," Ph.D. Thesis, Univ. of Derby, Derby, UK (2002).

3. J. Hasegawa, T.-Y. Hwang, H.-C. Kim, D.-W. Kim, and M.-H. Choi, "Measurement-based objective metric for printer resolution," Proc. SPIE 6494, 64940D1-12 (2007).

4. Woonyoung Jang and Jan P. Allebach, "Characterization of printer mtf," Proc. SPIE 6059, 60590D (2006).

5. A. J. Lindner, N. Bonnier, C. Leynadier, and F. Schmitt, "Evaluation of characterization methods of printer MTF," Proc. SPIE $\mathbf{6 8 0 8}$ 680806 (2008)

6. ISO 12233, "ISO 12233:2000 photography-electronic still picture cameras-resolution measurements," (ISO) 2000.

7. ISO 16067-1, "ISO 16067-1:2003 photography-spatial resolution measurements of electronic scanners for photographic images-part 1: scanners for reflective media," (ISO) 2003.

8. J. Arney, P. Anderson, P. Mehta, and K. Ayer, "The MTF of a printing systems," Proc. IS\&T/SPIE, 1, pp. 367-369 (2000).

9. Y. Bang, S. H. Kim, and D. C. Choi, "Printer resolution measurement based on slanted edge method," Proc. SPIE 6808, 680807 (2008).

10. Nicolas Bonnier, "Contributions to spatial gamut mapping algorithms," Ph.D. thesis, Telecom Paris-Tech, Paris, France (2008).

11. C. E. Shannon, "Communication in the presence of noise," Proc. IRE 37, 10-21 (1949).

12. R. Ulichney, Digital Halftoning, MIT Press (1990).

13. M. Lejeune, Statistique-La Theorie et Ses Applications, Springer, Berlin (2005).

14. A. J. Lindner, N. Bonnier, C. Leynadier, and F. Schmitt, "Measurement of printer MTFs," Proc. SPIE 7240, 72420N (2009).

15. J. W. Coltman, "The specification of imaging properties by response to a sine wave input," J. Opt. Soc. Am. A 44, 468 (1954).

16. N. Bonnier, A. J. Lindner, C. Leynadier, and F. Schmitt, "Compensation of printer MTFs," Proc. SPIE 7241, 724119 (2009).

17. B. Keelan, Handbook of Image Quality: Characterization and Prediction, Marcel Dekker, New York (2002).

18. T. F. Chan and J. Shen, "Theory and computation of variational image deblurring," Tech. Rep. UCLA and Univ. of Minnesota (2007).

19. N. Wiener, Extrapolation, Interpolation, and Smoothing of Stationary Time Series, Wiley, New York (1949).

20. J. C. Russ, The Image Processing Handbook, CRC Press, Boca Raton, FL (2007)

21. A. J. Lindner, "Measurement and compensation of printer modulation transfer functions," Master's Thesis, Telecom Paristech and Univ. of Stuttgart (2007).

22. N. Bonnier, F. Schmitt, M. Hull, and C. Leynadier, "Spatial and color adaptive gamut mapping: a mathematical framework and two new algorithms," Proc. 15th IS\&T/SID Color Imag. Conf., pp. 267-272 (2007).

23. R. Kimmel, D. Shaked, M. Elad, and I. Sobel, "Space dependent color gamut mapping: A variational approach," IEEE Trans. Image Process. 14, pp. 796-803 (2005).

24. J. Morovič and Y. Wang, "A multi-resolution, full-colour spatial gamut mapping algorithm," Proc. 11th IS\&T/SID Color Imag. Conf., pp. 282-287 (2003).

25. R. Balasubramanian, R. de Queiroz, and R. Eschbach, "Gamut mapping to preserve spatial luminance variations," Eighth Color Imag. Conf. Color Sci. Eng. Syst. Technol. Appl. 1, pp. 122-128 (2000).

26. N. Bonnier and E. P. Simoncelli, "Locally adaptive multiscale contrast optimization," 12th IEEE Int. Conf. Image Process., pp. 949$952(2005)$

27. I. Farup, C. Gatta, and A. Rizzi, "A multiscale framework for spatial gamut mapping," IEEE Trans. Image Process. 10, pp. 2423-2435 (2007).

28. P. Zolliker and K. Simon, "Retaining local image information in global gamut mapping algorithms," IEEE Trans. Image Process. 16, pp. 664-672 (2007)

29. O. Kolas and I. Farup, "Efficient hue-preserving and edge-preserving spatial color gamut mapping," 15th IS\&T/SID Color Imag. Conf., 1, pp. 207-212 (2007).

30. C. Tomasi and R. Manduchi, "Bilateral filtering for gray and color images," 6th Int. Conf. Comput. Vis., IEEE, pp. 839-846 (1998).

31. E. H. Adelson, E. P. Simoncelli, and W. T. Freeman, "Pyramids and multiscale representations," Proc. European Conf. Visual Percep. Vol. 1, pp. 3-16 (1990)

32. P. Zolliker and K. Simon, "Adding local contrast to global gamut mapping algorithms," 3rd European Conf. Colour Graphics Imag. Vis., 1, pp. 257-261 (2006).

33. N. Bonnier, F. Schmitt, and C. Leynadier, "Improvements in spatial and color adaptive gamut mapping algorithms," 4th IS\&T/SPIE European Conf. Colour Graphics Imag. Vis., pp. 341-346 (2008).

34. ISO 3664, "ISO 3664:2000 Viewing conditions-Graphic technology and photography," ISO (2000).

35. N. Bonnier, F. Schmitt, and C. Leynadier, "Compensating the print- 
er's modulation transfer function in spatial and color adaptive rendering workflows," 16th IS\&T/SID Color Imag. Conf., Vol. 1, pp. 93-98 (2008).

36. ISO 12640-2, "ISO 12640-2:2004 Graphic technology-Prepress digital data exchange-Part 2: XYZ/sRGB encoded standard colour image data (XYZ/SCID)," ISO (2004).

37. ISO 12640-3, "ISO 12640-3:2007 Graphic technology-Prepress digital data exchange-Part 3: CIELAB standard colour image data (CIELAB/SCID)," ISO (2007).

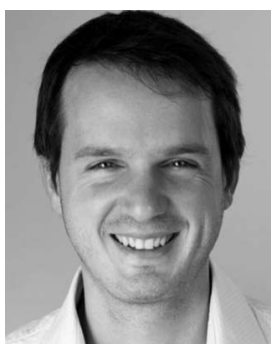

Nicolas Bonnier is the leading color scientist of a research team at Océ Print Logic Technologies. He represents Océ in the International Color Consortium. He is also a part-time lecturer at the ENS Louis Lumière (Paris) from which he graduated in 2000, majoring in photography. He received his Master's degree in electronic imaging from Université Pierre et Marie Curie (Paris) in 2001, then was a member of the Laboratory for Computational Vision with Professor Simoncelli at New York University from 2002 to 2005. He completed a PhD in signal and image processing from 2005 to 2008 under the direction of Professor Schmitt, Télécom Paristech, sponsored by Océ.

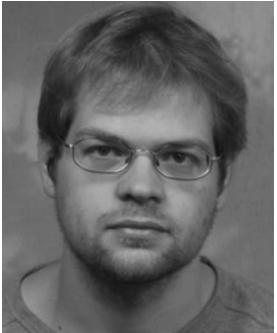

Albrecht $\mathrm{J}$. Lindner is a $\mathrm{PhD}$ student at EPFL (Lausanne, Switzerland). He is supervised by Professor Sabine Ssstrunk and is sponsored by Océ. He graduated from the University of Stuttgart (Germany) and Télécom Paristech (France) within a German-French exchange program in 2008. His two Master's degrees are in the domains of electrical engineering (Stuttgart) and signal and image processing (Paris). His Master's thesis was directed by Professor Francis Schmitt and Professor Joachim Speidel, sponsored by Océ. 